\title{
BMJ Open Surgical complications and their impact on patients' psychosocial well-being: a systematic review and meta-analysis
}

\author{
Anna Pinto, ${ }^{1}$ Omar Faiz, ${ }^{1}$ Rachel Davis, ${ }^{1}$ Alex Almoudaris, ${ }^{1}$ Charles Vincent ${ }^{2}$
}

To cite: Pinto A, Faiz 0, Davis $\mathrm{R}$, et al. Surgical complications and their impact on patients' psychosocial well-being: a systematic review and meta-analysis. BMJ Open 2016;6:e007224. doi:10.1136/bmjopen-2014007224

\section{- Prepublication history} and additional material is available. To view please visit the journal (http://dx.doi.org/ 10.1136/bmjopen-2014007224)

Received 16 November 2014 Revised 24 November 2015 Accepted 27 November 2015

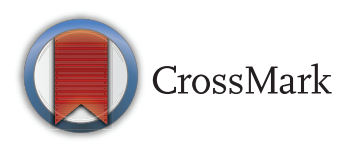

\footnotetext{
${ }^{1}$ Division of Surgery, Department of Surgery \& Cancer, Imperial College London, London, UK

${ }^{2}$ Department of Experimental Psychology, Oxford University, Oxford, UK
}

Correspondence to Dr Anna Pinto;

a.pinto@imperial.ac.uk

\section{ABSTRACT}

Objective: Surgical complications may affect patients psychologically due to challenges such as prolonged recovery or long-lasting disability. Psychological distress could further delay patients' recovery as stress delays wound healing and compromises immunity. This review investigates whether surgical complications adversely affect patients' postoperative well-being and the duration of this impact.

Methods: The primary data sources were 'PsychINFO', 'EMBASE' and 'MEDLINE' through OvidSP (year 2000 to May 2012). The reference lists of eligible articles were also reviewed. Studies were eligible if they measured the association of complications after major surgery from 4 surgical specialties (ie, cardiac, thoracic, gastrointestinal and vascular) with adult patients' postoperative psychosocial outcomes using validated tools or psychological assessment. 13605 articles were identified. 2 researchers independently extracted information from the included articles on study aims, participants' characteristics, study design, surgical procedures, surgical complications, psychosocial outcomes and findings. The studies were synthesised narratively (ie, using text). Supplementary metaanalyses of the impact of surgical complications on psychosocial outcomes were also conducted.

Results: 50 studies were included in the narrative synthesis. Two-thirds of the studies found that patients who suffered surgical complications had significantly worse postoperative psychosocial outcomes even after controlling for preoperative psychosocial outcomes, clinical and demographic factors. Half of the studies with significant findings reported significant adverse effects of complications on patient psychosocial outcomes at 12 months (or more) postsurgery. 3 supplementary meta-analyses were completed, 1 on anxiety (including 2 studies) and 2 on physical and mental quality of life (including 3 studies). The latter indicated statistically significantly lower physical and mental quality of life $(p<0.001)$ for patients who suffered surgical complications.

Conclusions: Surgical complications appear to be a significant and often long-term predictor of patient postoperative psychosocial outcomes. The results highlight the importance of attending to patients' psychological needs in the aftermath of surgical complications.

\section{Strengths and limitations of this study}

- This is, to our knowledge, the first systematic review of the literature assessing the impact of surgical complications on patients' psychosocial well-being.

- The validity of the findings is increased by the fact that only studies that used validated selfreport measures for the assessment of patients' well-being were included in the review, as well as by the use of a very comprehensive search strategy for the identification of relevant literature.

- Caution should be taken when interpreting these findings to other specialties as the review was limited in four surgical specialties.

- A limitation of this review was the very small number of studies with sufficient data for the quantitative synthesis, which did not also permit certain types of sensitivity analyses such as by surgical specialty or type of surgery.

\section{INTRODUCTION}

Surgical complications pose significant challenges for surgical patients. Complications may vary from very minor events that can be resolved relatively quickly without the need for pharmacological treatment or other intervention, to more serious events which can be life threatening, require multiple interventions (eg, return to theatre), delay patient's discharge and may lead to multiorgan failure or even death. ${ }^{1}$ A recent review of the literature found that postoperative complications contribute to increased mortality, length of stay and an increased level of care at discharge. ${ }^{2}$

Other than the complications' impact on patients' postoperative recovery, they may also affect patients psychologically. They may contribute to the experience of psychological distress such as depression or anxiety due to the challenges that are inherent to them in terms of prolonged recovery or long-lasting disability (eg, severe postoperative pain, permanent 
disfigurement). An early study found that patients who experienced serious adverse events after surgery reported higher levels of distress than people who had experienced serious accidents or bereavements and psychosocial adjustment worse than in patients with serious medical conditions. ${ }^{3}$ Moreover, the authors of an interview study on patients' experiences of cardiothoracic surgery reported that a small number of patients who had a long and complicated postoperative hospital stay expressed intense feelings of hopelessness and depression. ${ }^{4}$ Psychological distress resulting from the experience of surgical complications could further delay patients' recovery from surgery as increased levels of stress delay wound healing ${ }^{56}$ and compromise immunity. ${ }^{7-9}$

This review aims to critically review and synthesise the existing literature on the impact of surgical complications on adult surgical patients' psychosocial well-being and to estimate the duration of this impact. For the purpose of this review, psychosocial well-being was defined quite broadly including psychosocial outcomes of relevance to surgery such as anxiety, depression, quality of life (QoL) and post-traumatic stress. Quantitative studies which assessed the association of surgical complications with adult patients' psycho-social outcomes post-surgery were therefore reviewed. Our hypothesis was that the occurrence of surgical complications adversely affects patient psychosocial outcomes. Therefore, this systematic review aims to examine whether surgical complications impact adversely on patient psychosocial outcomes and the duration of this impact.

\section{METHODS}

\section{Search strategy}

The following databases were searched through OvidSP: 'PsychINFO' (1967 to 25 May 2012), 'EMBASE' (1947 to 25 May 2012) and 'MEDLINE' (1948 to 25 May 2012). A search strategy was developed specific to each database. The three facets of the search strategy were:

A. Adult surgical patients

Terms such as patients, inpatients, outpatients, men and women were used for this facet.

B. Patient psychosocial outcomes

A broad definition of psychosocial outcomes was considered for the purposes of this systemic review including search terms for anxiety, depression, QoL and post-traumatic stress. ${ }^{10}$ Two generic terms were also used, that is, well-being and emotions. The search did not include specific measures, instead it included terms for the outcomes specified above.

C. Surgical complications

Surgical complications were defined as any adverse event in relation to the surgical procedure including search terms for complications (eg, adverse events, untoward incidents) and terms about the surgical setting (eg, surgical, postoperative).

Each of the facets was expanded into a list of search terms truncated and combined with each other using
Boolean operators, and also by mapping those to their relevant MeSH headings and subheadings in each database (through explosion of each MeSH heading). The search was restricted to titles and abstracts, and the results were limited to studies that used human participants and were written in English. The search strategies are presented in online supplementary material 1 . Database searching was complemented by reviewing the reference lists of eligible articles.

\section{Eligibility criteria}

Studies were included in the review if they met the following criteria:

- Any quantitative study that measured the association of surgical complications with adult patients' psychosocial outcomes after surgery, either as a primary or secondary aim. Studies that measured surgical complications and psychosocial outcomes but not their association were not included as a primary analysis of reported data was beyond the scope of this review. Moreover, specific types of complications were not predefined as this review was interested in the impact of any surgical complications on patients' well-being.

- Psychosocial outcomes were measured with validated self-report tools or psychological assessment.

- Studies that reported surgical complications after cardiac, thoracic, gastrointestinal or vascular surgery, where complications are more likely to occur. ${ }^{11}$ Studies of neuropsychological complications (eg, delirium) and studies of transplantation procedures were excluded.

Conference proceedings, non-empirical data and articles that were published before the year 2000 or with the majority of their participants recruited before the year 2000 were excluded. This current approach in the selection of literature was expected to reduce bias resulting from studies of out-dated surgical practices.

\section{Study selection}

A total of $50 \%$ of the abstracts were reviewed independently by two researchers (AP and RD) and disagreements were resolved by consensus. The remaining half of the retrieved abstracts were reviewed by the primary researcher (AP) based on the consensus that was achieved for the first half. After excluding ineligible articles at abstract and title level, the remaining articles were assessed in full text. The eligibility criteria were applied again on each article. Reasons for exclusion were coded. Articles for which there was uncertainty were discussed between the primary researcher (AP), a researcher with background in psychology (RD) and a researcher with background in surgery (AA). Any disagreements were resolved by consensus.

\section{Data extraction and quality assessment}

The primary researcher (AP) and a researcher with a background in surgery (AA) independently extracted data from 20 articles, which they reviewed for any 
disagreements. Disagreements were resolved by consensus or referral to a third senior researcher (OF). Data were extracted from the remaining articles by the primary researcher and were later checked by the second reviewer (AA). A total of 10 authors were contacted by email to provide information that was not included in the manuscripts. Three articles were excluded from the analysis because their authors did not respond to our requests for further information. Information was extracted from each article on study aims, participants' characteristics, study design, surgical procedures, surgical complications (ie, types, definitions and method of recording, where available), psychosocial outcomes (ie, scales, and time points of measurement), and the association of psychosocial outcomes with surgical complications. The latter included any reported findings on the association of surgical complications with the psychosocial outcomes, including both overall scale and subscale scores where available.

The quality of the included studies was assessed with the Newcastle Ottawa Scales (NOS). ${ }^{12}$ The scales were modified in order to reflect the research questions of the review and to also incorporate the assessment of cross-sectional studies.

\section{Data synthesis}

The included studies were first synthesised narratively (ie, using words and text). In order to quantify the degree of the impact of surgical complications on psychosocial outcomes, quantitative procedures were also used. A meta-analysis was conducted on each extracted psychosocial outcome using Review Manager (V.5.2). ${ }^{13}$ $\mathrm{I}^{2}$ was used to calculate the heterogeneity present in the meta-analyses. Heterogeneity was considered low when it was below $25 \%$ and high above $50 \% .^{14}$ A random-effects approach was chosen, as a degree of heterogeneity between studies should always be assumed in social sciences. ${ }^{15}$ Where multiple assessments were conducted in one single study, only the one furthest from the participants' surgery was included in the meta-analysis.

\section{RESULTS}

In total, 18585 articles were retrieved in total across the three databases. After removing duplicate references, a total of 13605 papers were reviewed at abstract and title level. Nine hundred and ninety-four articles remained to be assessed in full text. A total of 51 articles (50 studies) were eligible for inclusion in the final stage of the review (see figure 1).

\section{Study characteristics}

Details of the included studies are presented in tables 1-3. A total of 28 studies were conducted in Europe, 14 in the USA, 3 in Australia, 2 in Turkey, 1 in Egypt, 1 in Japan and 1 in Taiwan. There were 29 studies in gastrointestinal, ${ }^{16-44}$ 17 in cardiothoracic ${ }^{45-62}$ and 4 in vascular surgery. ${ }^{63-66}$
The majority of the included studies (40 studies) assessed major procedures. The most common indications for surgery were heart conditions, followed by different types of cancer. Twenty-three studies examined the association between surgical complications and patients' well-being as a primary research aim. ${ }^{17} 1928$ 30-38 $43474850-5355626466$ The remaining examined this relationship as part of an exploration of the association of different clinical factors with patients' postoperative well-being. The majority of the studies were cohort studies. There were four case-control and 20 cross-sectional studies. The majority of the studies were prospective, including baseline measures of psychosocial outcomes.

QoL was the main reported psychosocial outcome. Three studies measured anxiety, ${ }^{30} 4062$ four studies measured depression ${ }^{31} 414962$ and one study measured mood states. ${ }^{41}$ No other psychosocial outcomes were measured. The Short Form Health Survey (SF)-36 (and its associated versions, ie, SF-12, SF-20) was the most commonly used scale for the measurement of QoL. ${ }^{18}$ 25-31 36-38 $424345464851-55$ 57-59 6163

The vast majority of the studies used a priori definitions of complications. For example, Bloemen et $a l^{19}$ recorded only severe complications based on a grading system of surgical complications. Dasgupta et $a l^{23}$ also recorded major complications which were defined as "those associated with systemic illness requiring transfer to a higher level of care or requiring relaparotomy, or complications needing interventional radiology". Others used predefined categories of complications such as infections, respiratory complications, chronic postoperative pain or perioperative myocardial infarctions. A total of 14 studies did not define or describe the complications that were recorded. The majority of the studies recorded a range of postoperative complications. Eighteen studies focused on a single category of complications (eg, anastomotic leaks, perioperative myocardial infarctions, wound complications, atrial fibrillation). Complications were mostly recorded through medical records review, clinical examinations and review of administrative databases.

Study quality varied. The scores of the included studies ranged from 2 to 8 , with a mean score of 5.9. Points were deducted for the following reasons: lack of information on how complications were defined or on the methods for their recording, ${ }^{16-18}$ 21-23 $2529353740-$

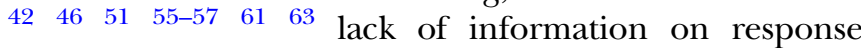
rates, ${ }^{16} 212225-27 \quad 29 \quad 3740 \quad 5052 \quad 545557 \quad 60 \quad 61$ baseline psychosocial outcomes were either not measured or controlled for, ${ }^{17} 1920252730-36 \quad 38-40 \quad 43-4547495363$ and demographic or clinical factors were not controlled for. ${ }^{20} 252731323440434551566163$ Seven studies scored exceptionally low (ie, below 4 ).

\section{The impact of surgical complications on patients'} well-being

The majority of studies $(n=32)$ found that patients who suffered surgical complications had significantly worse 

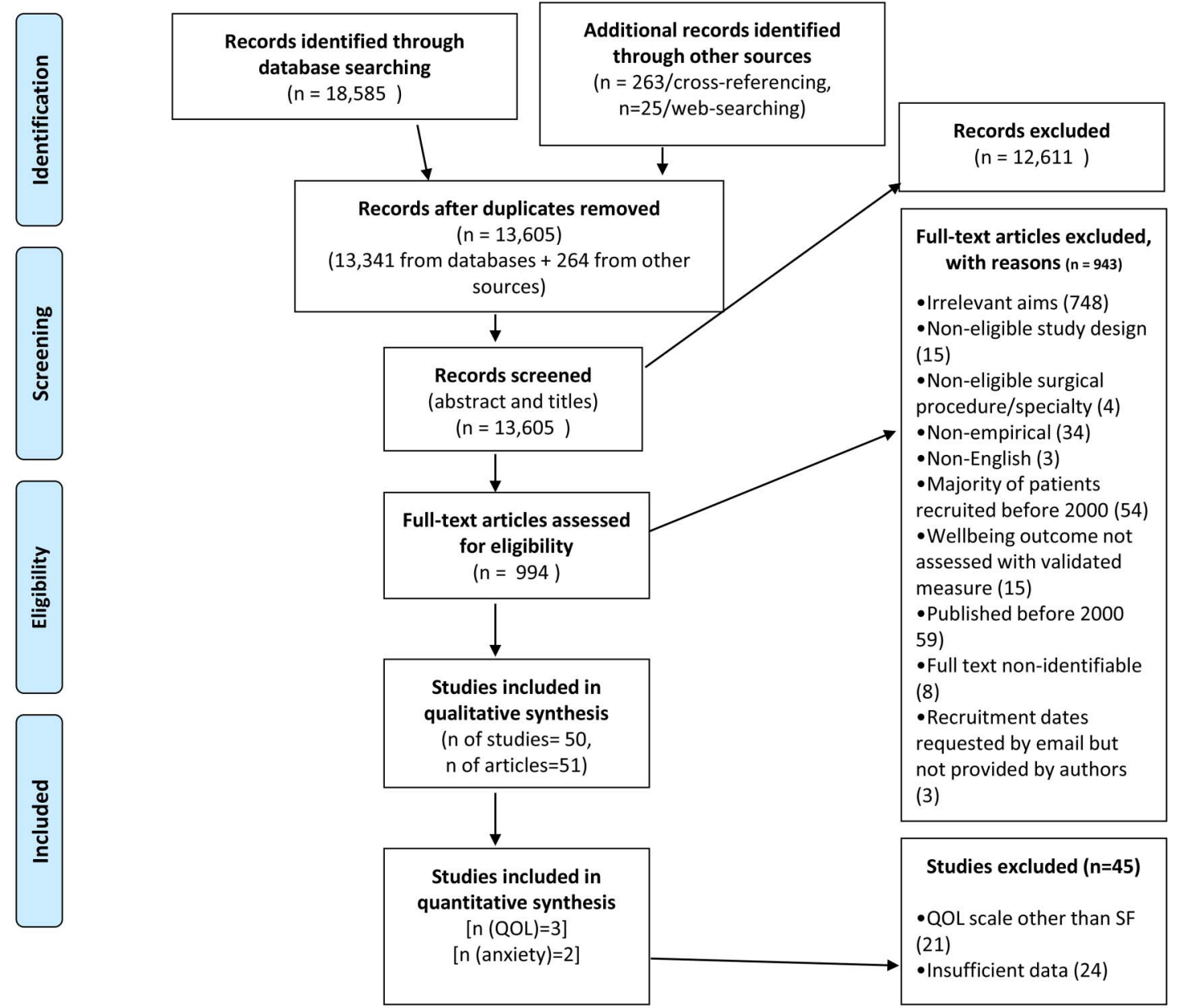

Figure 1 PRISMA diagram. SF, Short Form Health Survey; QOL, quality of life.

postoperative psychosocial outcomes than patients with uncomplicated recovery. ${ }^{16-20} 22 \quad 24 \quad 25 \quad 28 \quad 30 \quad 31 \quad 33 \quad 35-37 \quad 39$ $41-48 \quad 50-52 \quad 54 \quad 57 \quad 60 \quad 6265$

major surgical procedures but also after relatively minor operations such as hernia repairs. ${ }^{18} 28303143$ The vast majority $(n=25,78 \%)$ were of high quality (ie, quality assessment score greater than 6 out of 8 ). For instance, more than half of the studies with significant findings had measured and controlled for patients' baseline psychosocial outcomes $(n=18)^{16} 18222428374142464850-52$ $\begin{array}{lllllll}54 & 57 & 60 & 62 & 65 & \text { and used multivariate analyses }\end{array}$ $(\mathrm{n}=21), 1_{16}^{16} 18 \quad 19 \begin{array}{lllllllllllllll}24 & 24 & 25 & 28 & 35 & 37 & 39 & 41 & 42 & 44 & 46 & 47 & 50 & 52\end{array}$

54606265 suggesting that complications remained a significant independent predictor of patients' postoperative well-being even after controlling for a range of clinical and demographic factors. Psychosocial outcomes that were significantly negatively affected by surgical complications included physical, emotional and social aspects of patients' QoL as well as anxiety and depression levels (see table 4). Complications that were found to be significantly associated with worse psychosocial outcomes included both major events such as perioperative myocardial infarctions after $\mathrm{CABG}^{50}{ }^{50}$ severe incontinence after internal sphincterectomy ${ }^{31}$ or graft-related events after vascular surgery, ${ }^{65}$ and minor complications such as wound infections after hepatic resection, ${ }^{20}$ or new cardiac arrhythmias after CABG. ${ }^{54}$ The complications that were significantly associated with patients' postoperative psychosocial outcomes are presented in tables 1-3.

Six studies reported a confounding association between surgical complications and patients' well-being (ie, complications were significantly associated with worse psychosocial outcomes only under certain conditions) ${ }^{21} 3240$ or complications were significantly associated with psychosocial outcomes at univariate but not at multivariate analysis. ${ }^{4959}{ }^{64}$ A total of 12 studies did not find a significant association of surgical complications with postoperative psychosocial outcomes. ${ }^{23} 2627293438535556616366$ The majority of them $(n=7)$ scored below 6 on quality assessment. For example, four studies had very small samples. ${ }^{26} 273438$

\section{Meta-analyses}

A series of supplementary meta-analyses were attempted on each extracted psychosocial outcome (ie, QoL, anxiety, depression). For a meta-analysis on QoL, a synthesis of data from widely disparate assessment 


\begin{tabular}{|c|c|c|c|c|c|c|c|c|c|c|c|c|}
\hline $\begin{array}{l}\text { First } \\
\text { author's } \\
\text { name }\end{array}$ & Year & Country & $\begin{array}{l}\text { Primary or } \\
\text { Secondary } \\
\text { aim }\end{array}$ & $\begin{array}{l}\text { Sample (N=number } \\
\text { of patients in } \\
\text { analysis/eligible } \\
\text { patients, Nt(i) } \\
\text { =sample size per } \\
\text { time point, } \\
\mathrm{Nc}=\text { patients with } \\
\text { complications, } \\
\mathrm{N} 1=\text { cases vs } \\
\mathrm{N} 2=\text { controls) }\end{array}$ & $\begin{array}{l}\text { Patient inclusion } \\
\text { criteria }\end{array}$ & Study design & Type of surgery & $\begin{array}{l}\text { Surgical complications/ } \\
\text { method of recording }\end{array}$ & $\begin{array}{l}\text { Psychosocial } \\
\text { outcome/time } \\
\text { points/ } \\
\text { measurement tool }\end{array}$ & $\begin{array}{l}\text { Significant } \\
\text { association of } \\
\text { surgical } \\
\text { complications } \\
\text { with patients' } \\
\text { well-being } \\
\text { (yes/no/ } \\
\text { confounding) }\end{array}$ & $\begin{array}{l}\text { Types of } \\
\text { complications } \\
\text { and time points } \\
\text { of significant } \\
\text { effects }\end{array}$ & $\begin{array}{l}\text { Quality } \\
\text { assessment } \\
\text { score (out } \\
\text { of } 8 \text { ) }\end{array}$ \\
\hline Anthony & 2003 & US & Secondary & $\begin{array}{l}\mathrm{Nt} 1=71 / ? \\
\mathrm{Nt} 2=63 \\
\mathrm{Nc}=16\end{array}$ & $\begin{array}{l}\text { Colorectal cancer, } \\
\text { male patients who } \\
\text { underwent open } \\
\text { surgical therapy }\end{array}$ & $\begin{array}{l}\text { Observational, } \\
\text { cohort, } \\
\text { prospective }\end{array}$ & $\begin{array}{l}\text { Open surgical } \\
\text { therapy for } \\
\text { colorectal cancer }\end{array}$ & $\begin{array}{l}\text { Morbidity was defined as any } \\
\text { event that resulted in the } \\
\text { need for additional therapy or } \\
\text { readmission to the hospital } \\
\text { within } 30 \text { days of initial } \\
\text { discharge/method not } \\
\text { specified }\end{array}$ & $\begin{array}{l}\text { QoL/at time of } \\
\text { diagnosis and } \\
12 \text { months after } \\
\text { surgery/FACT-C }\end{array}$ & Yes* $^{*}$ & $\begin{array}{l}\text { Any } \\
\text { complications/ } \\
12 \text { months } \\
\text { postsurgery }\end{array}$ & 6 \\
\hline Avery & 2006 & UK & Primary & $\begin{array}{l}\mathrm{N}=139 / 162 \\
\mathrm{Nc}=37\end{array}$ & $\begin{array}{l}\text { Patients with } \\
\text { oesophageal or } \\
\text { gastric cancer who } \\
\text { underwent upper } \\
\text { gastrointestinal } \\
\text { surgical treatment }\end{array}$ & $\begin{array}{l}\text { Observational, } \\
\text { cross-sectional }\end{array}$ & $\begin{array}{l}\text { Upper } \\
\text { gastrointestinal } \\
\text { surgical treatment } \\
\text { for oesophageal } \\
\text { or gastric cancer }\end{array}$ & $\begin{array}{l}\text { A major complication was } \\
\text { defined as reoperation, } \\
\text { readmission to the high } \\
\text { dependency or intensive } \\
\text { care unit, readmission to the } \\
\text { hospital within } 30 \text { days of } \\
\text { operation, or death within } \\
30 \text { days of operation or later } \\
\text { if the patient did not leave } \\
\text { the hospital/method not } \\
\text { specified }\end{array}$ & $\begin{array}{l}\text { QOL/39.6 days after } \\
\text { treatment (range 6- } \\
\text { 105)/EORTC } \\
\text { QLQ-C30 }\end{array}$ & Yes & $\begin{array}{l}\text { Any } \\
\text { complications/ } \\
39.6 \text { days after } \\
\text { treatment (range } \\
6-105 \text { ) }\end{array}$ & 5 \\
\hline Bitzer & 2008 & Germany & Secondary & $\begin{array}{l}\mathrm{Nt} 1=151 / 205 \\
\mathrm{Nt} 2=130(86.1 \%) \\
\mathrm{Nc}(\text { complaints)=49 } \\
\mathrm{Nc}(\text { wound infection) } \\
=5 \\
\mathrm{Nc}(\text { seroma })=13 \\
\mathrm{Nc} \text { (pneumonia) }=1 \\
\mathrm{Nc} \text { (other)=28 }\end{array}$ & $\begin{array}{l}\text { Patients undergoing } \\
\text { cholecystectomy }\end{array}$ & $\begin{array}{l}\text { Observational, } \\
\text { cohort, } \\
\text { prospective }\end{array}$ & Cholecystectomy & $\begin{array}{l}\text { Retrospective list: any } \\
\text { complaint, wound infection, } \\
\text { seroma, pneumonia, other } \\
\text { complaints/patient reports }\end{array}$ & $\begin{array}{l}\text { QoL/14 days } \\
\text { preoperative, } 14 \text { days } \\
\text { postoperative, and } \\
6 \text { months } \\
\text { postoperative/SF-36 }\end{array}$ & Yes* & $\begin{array}{l}\text { Any } \\
\text { complications/ } \\
6 \text { months } \\
\text { postsurgery }\end{array}$ & 7 \\
\hline Bloemen & 2009 & $\begin{array}{l}\text { The } \\
\text { Netherlands }\end{array}$ & Primary & $\begin{array}{l}\mathrm{N}=121 / 170 \\
\mathrm{NC}=33\end{array}$ & $\begin{array}{l}\text { Patients with rectal } \\
\text { cancer }\end{array}$ & $\begin{array}{l}\text { Observational, } \\
\text { cross-sectional }\end{array}$ & $\begin{array}{l}\text { Surgical } \\
\text { treatment for } \\
\text { adenocarcinoma } \\
\text { of the rectum }\end{array}$ & $\begin{array}{l}\text { Only severe complications } \\
\text { were considered: grade III or } \\
\text { IV complications (according } \\
\text { to Dindo's model) were } \\
\text { defined as severe, whereas } \\
\text { absence of complications or } \\
\text { grade I and II complications } \\
\text { were defined as absent or } \\
\text { mild complications/patient } \\
\text { records }\end{array}$ & $\begin{array}{l}\text { QoL/36 (16-51) } \\
\text { months } \\
\text { postoperative/ } \\
\text { EORTC QLQ-C30 } \\
\text { and CR38 }\end{array}$ & Yes & $\begin{array}{l}\text { Severe } \\
\text { postoperative } \\
\text { complications/ } \\
\text { median of } 36 \\
\text { (range 16-51) } \\
\text { months } \\
\text { postsurgery }\end{array}$ & 6 \\
\hline Bruns & 2010 & Germany & Secondary & $\begin{array}{l}\mathrm{N}=96 / 188 \\
\mathrm{Nc}(\text { any morbidity) } \\
=30 \\
\mathrm{Nc} \text { (wound } \\
\text { infections) }=10\end{array}$ & $\begin{array}{l}\text { Patients who } \\
\text { underwent curative } \\
\text { hepatic resection for } \\
\text { malignant or } \\
\text { non-malignant } \\
\text { diseases, disease } \\
\text { free at time of } \\
\text { assessment }\end{array}$ & $\begin{array}{l}\text { Observational, } \\
\text { cross-sectional }\end{array}$ & Hepatectomy & $\begin{array}{l}\text { Surgical (eg, bile leak or } \\
\text { biloma, pneumothorax, } \\
\text { wound infection, liver } \\
\text { abscess, bleeding, and } \\
\text { surgical dehiscence) and } \\
\text { medical (eg, pleural effusion, } \\
\text { renal failure, hepatic failure, } \\
\text { pneumonia, cardiac } \\
\text { insufficiency and cholangitis)/ } \\
\text { patient records }\end{array}$ & $\begin{array}{l}\text { QoL/ 3-36 months } \\
\text { postoperative /SF-12 }\end{array}$ & Yes & $\begin{array}{l}\text { Wound } \\
\text { infections/3- } \\
36 \text { months } \\
\text { postsurgery }\end{array}$ & 5 \\
\hline
\end{tabular}

of patients in

(i)

me point

First

Pecondary $\mathrm{N}$-cases

olorectal cance

underwent open

cohort

Open surgical

$\mathrm{Nt} 2=63$

Patients with

esophageal or

(ancer who

astrointestinal

6 days after

eatment (rang

$\mathrm{Nc}$ (complaints) $=49$

$\mathrm{Nc}$ (wound infection)

$\mathrm{Nc}($ seroma $)=13$

$\mathrm{N}=121 / 170$

Patients with rectal

nal, Surgical

treatment for

Surgical (eg, bile leak or

biloma, pneumothorax, 

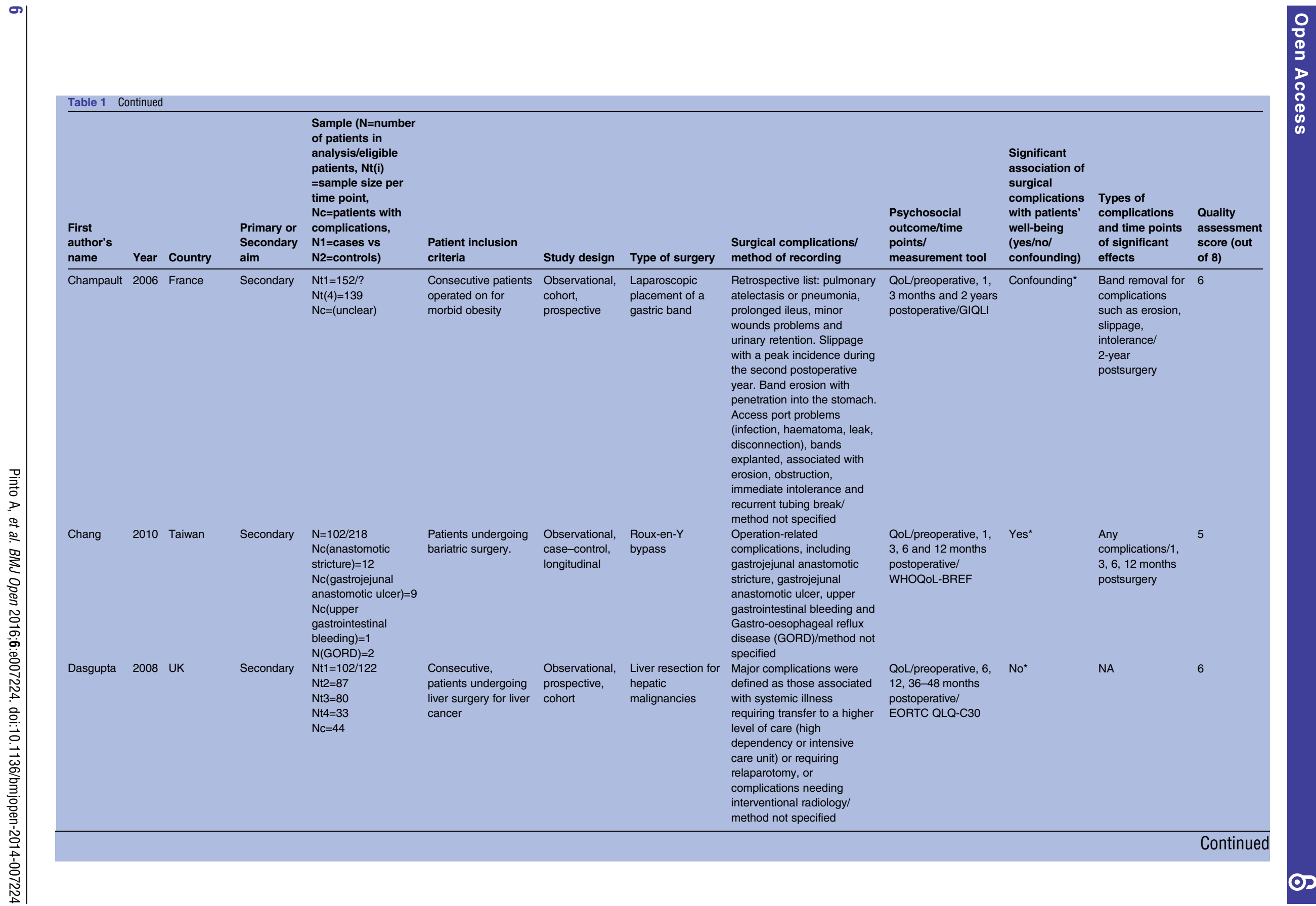

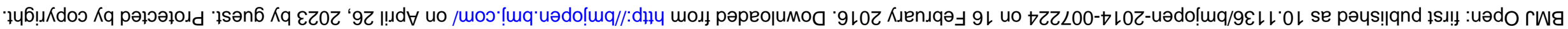




\begin{tabular}{|c|c|c|c|c|c|c|c|c|c|c|c|c|}
\hline $\begin{array}{l}\text { First } \\
\text { author's } \\
\text { name }\end{array}$ & Year & Country & $\begin{array}{l}\text { Primary or } \\
\text { Secondary } \\
\text { aim }\end{array}$ & $\begin{array}{l}\text { Sample ( } \mathrm{N}=\text { number } \\
\text { of patients in } \\
\text { analysis/eligible } \\
\text { patients, } \mathrm{Nt}(\mathrm{i}) \\
\text { =sample size per } \\
\text { time point, } \\
\mathrm{Nc=patients} \mathrm{with} \\
\text { complications, } \\
\mathrm{N} 1=\text { cases vs } \\
\mathrm{N} 2=\text { controls) }\end{array}$ & $\begin{array}{l}\text { Patient inclusion } \\
\text { criteria }\end{array}$ & Study design & Type of surgery & $\begin{array}{l}\text { Surgical complications/ } \\
\text { method of recording }\end{array}$ & $\begin{array}{l}\text { Psychosocial } \\
\text { outcome/time } \\
\text { points/ } \\
\text { measurement tool }\end{array}$ & $\begin{array}{l}\text { Significant } \\
\text { association of } \\
\text { surgical } \\
\text { complications } \\
\text { with patients' } \\
\text { well-being } \\
\text { (yes/no/ } \\
\text { confounding) }\end{array}$ & $\begin{array}{l}\text { Types of } \\
\text { complications } \\
\text { and time points } \\
\text { of significant } \\
\text { effects }\end{array}$ & $\begin{array}{l}\text { Quality } \\
\text { assessment } \\
\text { score (out } \\
\text { of 8) }\end{array}$ \\
\hline Delaney & 2003 & USA & Secondary & $\begin{array}{l}\mathrm{Nt} 1=109 / 109 \\
\mathrm{Nt} 2=82 / 109 \\
\mathrm{Nc}(\text { any) }=19 \\
\mathrm{Nc}(\text { major) }=9\end{array}$ & $\begin{array}{l}\text { Patients with } \\
\text { Crohn's disease }\end{array}$ & $\begin{array}{l}\text { Observational, } \\
\text { cohort, } \\
\text { prospective }\end{array}$ & $\begin{array}{l}\text { Surgery for CD } \\
\text { (abdominal } \\
\text { perineal, loop or } \\
\text { end stoma) }\end{array}$ & $\begin{array}{l}\text { Retrospectively listed } \\
\text { complications: anastomotic } \\
\text { leak, intra-abdominal } \\
\text { abscess, bleeding, venous } \\
\text { thrombosis, renal failure, and } \\
\text { pneumonia, dehydration, } \\
\text { intra-abdominal abscess, } \\
\text { small bowel obstruction and } \\
\text { wound infection/database } \\
\text { review }\end{array}$ & $\begin{array}{l}\text { QoL/preoperative and } \\
30 \text { days } \\
\text { postoperative/CGQL }\end{array}$ & Yes* & $\begin{array}{l}\text { Any } \\
\text { complications/ } \\
30 \text { days } \\
\text { postoperative }\end{array}$ & 7 \\
\hline Douma & 2011 & $\begin{array}{l}\text { The } \\
\text { Netherlands }\end{array}$ & Secondary & $\begin{array}{l}\mathrm{N}=296 / ? \\
\mathrm{Nc}=?\end{array}$ & $\begin{array}{l}296 \text { patients with } \\
\text { FAP who had been } \\
\text { surgically treated }\end{array}$ & $\begin{array}{l}\text { Observational, } \\
\text { cross-sectional }\end{array}$ & Surgery for FAP & $\begin{array}{l}\text { Surgery-related } \\
\text { complications/self-reports } \\
\text { +medical records }\end{array}$ & $\begin{array}{l}\text { QoL/0 to }>10 \text { years } \\
\text { postoperative/SF-36, } \\
\text { EORTC-QLQ-C38, } \\
\text { Social Functioning } \\
\text { subscale of the } \\
\text { Dutch version of } \\
\text { IBDQ }\end{array}$ & Yes & $\begin{array}{l}\text { Any } \\
\text { complications } / 0 \\
\text { to }>10 \text { years } \\
\text { postsurgery }\end{array}$ & 2 \\
\hline Dubernard & 2006 & France & Secondary & $\begin{array}{l}\mathrm{N} t 1=58 / ? \\
\mathrm{~N} 2=58 \\
\mathrm{Nc}=9\end{array}$ & $\begin{array}{l}\text { Women with } \\
\text { colorectal } \\
\text { endometriosis who } \\
\text { underwent a } \\
\text { segmental colorectal } \\
\text { resection }\end{array}$ & $\begin{array}{l}\text { Observational, } \\
\text { cohort, } \\
\text { prospective }\end{array}$ & $\begin{array}{l}\text { Laparoscopic } \\
\text { segmental } \\
\text { colorectal } \\
\text { resection for } \\
\text { endometriosis }\end{array}$ & $\begin{array}{l}\text { Retrospectively listed } \\
\text { complications: rectovaginal } \\
\text { fistulae, vessel injury of the } \\
\text { protective colostomy treated } \\
\text { by laparoscopic coagulation, } \\
\text { uroperitoneum requiring a } \\
\text { ureteral stent for } 6 \text { weeks } \\
\text { and an abscess behind } \\
\text { colorectal anastomosis } \\
\text { requiring a laparoscopic } \\
\text { drainage/patient } \\
\text { observations }\end{array}$ & $\begin{array}{l}\text { QoL/preoperative and } \\
\text { postoperative/SF-36 }\end{array}$ & $\mathrm{No}^{*}$ & NA & 6 \\
\hline El-Awady & 2009 & Egypt & Secondary & $\begin{array}{l}\mathrm{N}=40 / ? \\
\mathrm{NC}=14\end{array}$ & $\begin{array}{l}\text { Patients with } \\
\text { inguinal hernia }\end{array}$ & $\begin{array}{l}\text { Observational, } \\
\text { prospective, } \\
\text { cohort }\end{array}$ & $\begin{array}{l}\text { Anterior open } \\
\text { Lichtenstein } \\
\text { tension-free } \\
\text { hernioplasty }\end{array}$ & $\begin{array}{l}\text { Postoperative complications: } \\
\text { seroma, haematoma, } \\
\text { secondary infection, } \\
\text { neuralgia and anaesthesia/ } \\
\text { patient observations }\end{array}$ & $\begin{array}{l}\text { QoL/preoperative, } 3 \text {, } \\
6 \text { and } 12 \text { months } \\
\text { postoperative/SF-36 }\end{array}$ & No & NA & 4 \\
\hline Hawn & 2006 & USA & Primary & $\begin{array}{l}\mathrm{Nt} 1=1983 / 3518 \\
\mathrm{Nt} 2=1526(77 \%) \\
\mathrm{Nt3}=1603(81 \%) \\
\mathrm{Nc}(\text { neuralgia } \mathrm{t} 1)=94 \\
\mathrm{Nc}(\text { haematoma } \mathrm{t} 1) \\
=51 \\
\mathrm{Nc} \text { (orchitis } \mathrm{t} 1)=13 \\
\mathrm{Nc}(\text { recurrence } \mathrm{t} 1 \text { ) } \\
=76 \\
\mathrm{Nc}(\text { other } \mathrm{t} 1)=124\end{array}$ & $\begin{array}{l}\text { Men who received a } \\
\text { hernia repair }\end{array}$ & $\begin{array}{l}\text { Observational, } \\
\text { cohort, } \\
\text { prospective }\end{array}$ & $\begin{array}{l}\text { Inguinal } \\
\text { herniorrhaphy }\end{array}$ & $\begin{array}{l}\text { Complications were } \\
\text { summarised by } 4 \text { categories: } \\
\text { (1) haematoma/seroma, (2) } \\
\text { orchitis, (3) neuralgia of the } \\
\text { leg or groin, and (4) other. } \\
\text { complications classified as } \\
\text { 'other' included (1) early } \\
\text { postoperative complications } \\
\text { (urinary tract infection, } \\
\text { urinary retention, and }\end{array}$ & $\begin{array}{l}\text { QOL/pre-op, } 1 \\
\& 2 \text { years post-op/ } \\
\text { SF-36 }\end{array}$ & Yes $^{*}$ & $\begin{array}{l}\text { Neuralgia, } \\
\text { orchitis/2 years } \\
\text { postsurgery }\end{array}$ & 8 \\
\hline
\end{tabular}




\begin{tabular}{|c|c|c|c|c|c|c|c|c|c|c|c|c|}
\hline $\begin{array}{l}\text { First } \\
\text { author's } \\
\text { name }\end{array}$ & Year & Country & $\begin{array}{l}\text { Primary or } \\
\text { Secondary } \\
\text { aim }\end{array}$ & $\begin{array}{l}\text { Sample (N=number } \\
\text { of patients in } \\
\text { analysis/eligible } \\
\text { patients, Nt(i) } \\
\text { =sample size per } \\
\text { time point, } \\
\text { Nc=patients with } \\
\text { complications, } \\
\mathrm{N} 1=\text { cases vs } \\
\mathrm{N} 2=\text { controls) }\end{array}$ & $\begin{array}{l}\text { Patient inclusion } \\
\text { criteria }\end{array}$ & Study design & Type of surgery & $\begin{array}{l}\text { Surgical complications/ } \\
\text { method of recording }\end{array}$ & $\begin{array}{l}\text { Psychosocial } \\
\text { outcome/time } \\
\text { points/ } \\
\text { measurement tool }\end{array}$ & $\begin{array}{l}\text { Significant } \\
\text { association of } \\
\text { surgical } \\
\text { complications } \\
\text { with patients' } \\
\text { well-being } \\
\text { (yes/no/ } \\
\text { confounding) }\end{array}$ & $\begin{array}{l}\text { Types of } \\
\text { complications } \\
\text { and time points } \\
\text { of significant } \\
\text { effects }\end{array}$ & $\begin{array}{l}\text { Quality } \\
\text { assessment } \\
\text { score (out } \\
\text { of } 8 \text { ) }\end{array}$ \\
\hline & & & & $\begin{array}{l}\text { Nc(neuralgia } \mathrm{t} 2) \\
=105 \\
\mathrm{Nc}(\text { haematoma } \mathrm{t} 2) \\
=55 \\
\mathrm{Nc}(\text { orchitis } \mathrm{t} 2)=18 \\
\mathrm{Nc}(\text { other } \mathrm{t} 2)=150\end{array}$ & & & & $\begin{array}{l}\text { haematuria); (2) } \\
\text { life-threatening complications } \\
\text { (respiratory insufficiency, } \\
\text { myocardial ischaemia, } \\
\text { cardiac arrhythmia, } \\
\text { intraoperative hypotension } \\
\text { and stroke); and (3) } \\
\text { long-term complications } \\
\text { ( } 4 \text { weeks or more } \\
\text { postoperative)/patient reports } \\
\text { for neuralgia and orchitis + } \\
\text { expert consensus for } \\
\text { life-threatening complications }\end{array}$ & & & & \\
\hline Ince & 2011 & USA & Secondary & $\begin{array}{l}\mathrm{N} t 1=? / 568 \\
\mathrm{Nt2}=166 \\
\mathrm{Nc}=?\end{array}$ & $\begin{array}{l}\text { Patients who } \\
\text { underwent colorectal } \\
\text { resection for benign } \\
\text { and malignant } \\
\text { diseases. }\end{array}$ & $\begin{array}{l}\text { Observational, } \\
\text { cohort, } \\
\text { retrospective }\end{array}$ & $\begin{array}{l}\text { Laparoscopic } \\
\text { colorectal } \\
\text { resection }\end{array}$ & No reference & $\begin{array}{l}\text { QOL/pre-op, } 4 \text { weeks } \\
\text { post-op/SF-36 }\end{array}$ & $\mathrm{No}^{*}$ & NA & 3 \\
\hline Kalliomaki & 2009 & Sweden & Primary & $\begin{array}{l}\mathrm{N}(\text { total) }=184 / 423 \\
\mathrm{N} 1=92 \text { (cases) } \\
\mathrm{N} 2=92 \text { (controls) }\end{array}$ & $\begin{array}{l}\text { Patients who had } \\
\text { been operated on for } \\
\text { groin hernia. } \\
\text { Controls matched for } \\
\text { age, gender and } \\
\text { method of surgical } \\
\text { repair were allotted } \\
\text { from the group of } \\
\text { persons without } \\
\text { persisting pain } \\
\text { (grade } 1 \text { in IPQ) }\end{array}$ & $\begin{array}{l}\text { Observational, } \\
\text { case-control, } \\
\text { cross-sectional }\end{array}$ & Hernia repair & $\begin{array}{l}\text { Persistent postoperative pain } \\
\text { (patients with pain of grade } \\
3 \text {, ie, pain that could not be } \\
\text { ignored but did not interfere } \\
\text { with everyday activities, or } \\
\text { higher on IPQ)/patient } \\
\text { reports (IPQ) and clinical } \\
\text { examination }\end{array}$ & $\begin{array}{l}\text { QoL, anxiety, } \\
\text { depression/(on } \\
\text { average } 4.9 \text { years } \\
\text { postoperative, range } \\
>7 \text { years)/SF-36, } \\
\text { HADS }\end{array}$ & Yes & $\begin{array}{l}\text { Persistent } \\
\text { postoperative/ } \\
\text { mean of } \\
4.9 \text { years } \\
\text { postsurgery }\end{array}$ & 5 \\
\hline Kement & 2011 & Turkey & Primary & $\begin{array}{l}\mathrm{N}=253 / 351 \\
\mathrm{~N} \text { (incontinence) }=28 \\
\mathrm{~N}(\text { severe } \\
\text { incontinence) }=9 \\
\mathrm{~N}(\text { mild incontinence) } \\
=19\end{array}$ & $\begin{array}{l}\text { Consecutive patients } \\
\text { with chronic anal } \\
\text { fissure who } \\
\text { underwent open } \\
\text { lateral internal } \\
\text { sphincterotomy } \\
\text { (LIS). }\end{array}$ & $\begin{array}{l}\text { Observational, } \\
\text { cross-sectional }\end{array}$ & $\begin{array}{l}\text { Open lateral } \\
\text { internal } \\
\text { sphincterotomy }\end{array}$ & $\begin{array}{l}\text { Anal incontinence/patient } \\
\text { reports: WIS system + } \\
\text { clinical examination }\end{array}$ & $\begin{array}{l}\text { QoL/23.3 } \pm 7.1 \text { months } \\
\text { postoperative/SF-36 }\end{array}$ & Yes & $\begin{array}{l}\text { Severe } \\
\text { incontinence/23.3 } \\
(\mathrm{SD} \pm 7.1) \text { months } \\
\text { postsurgery }\end{array}$ & 5 \\
\hline Lim & 2006 & UK & Primary & $\begin{array}{l}\mathrm{N}=92 / 112 \\
\mathrm{Nc}(\text { leaks })=23 \\
\mathrm{Nc}(\text { clinical leaks) }=13 \\
\mathrm{Nc}(\text { subclinical leaks) } \\
=10\end{array}$ & $\begin{array}{l}\text { Consecutive patients } \\
\text { under the care of } \\
\text { three consultant } \\
\text { surgeons who } \\
\text { underwent } \\
\text { procedures with LRA }\end{array}$ & $\begin{array}{l}\text { Observational, } \\
\text { cross-sectional }\end{array}$ & LRA & $\begin{array}{l}\text { Anastomotic leaks (clinical } \\
\text { and subclinical)/patient } \\
\text { observations, CT scans, } \\
\text { Wireless Capsule Endoscopy } \\
\text { (WCE) }\end{array}$ & $\begin{array}{l}\text { QoL/10-18 months } \\
\text { postoperative/ } \\
\text { EORTC QoL }\end{array}$ & Confounding & $\begin{array}{l}\text { Anastomotic } \\
\text { leaks } / 10- \\
18 \text { months } \\
\text { postoperative }\end{array}$ & 5 \\
\hline & & & & & & & & & & & & Continued \\
\hline
\end{tabular}

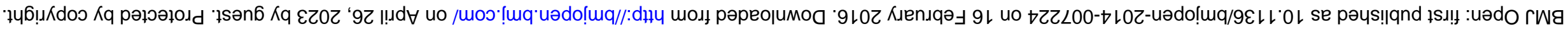




\begin{tabular}{|c|c|c|c|c|c|c|c|c|c|c|c|c|}
\hline $\begin{array}{l}\text { First } \\
\text { author's } \\
\text { name }\end{array}$ & Year & Country & $\begin{array}{l}\text { Primary or } \\
\text { Secondary } \\
\text { aim }\end{array}$ & $\begin{array}{l}\text { Sample ( } \mathrm{N}=\text { number } \\
\text { of patients in } \\
\text { analysis/eligible } \\
\text { patients, Nt(i) } \\
\text { =sample size per } \\
\text { time point, } \\
\mathrm{Nc}=\text { patients with } \\
\text { complications, } \\
\mathrm{N} 1=\text { cases vs } \\
\mathrm{N} 2=\text { controls) }\end{array}$ & $\begin{array}{l}\text { Patient inclusion } \\
\text { criteria }\end{array}$ & Study design & Type of surgery & $\begin{array}{l}\text { Surgical complications/ } \\
\text { method of recording }\end{array}$ & $\begin{array}{l}\text { Psychosocial } \\
\text { outcome/time } \\
\text { points/ } \\
\text { measurement tool }\end{array}$ & $\begin{array}{l}\text { Significant } \\
\text { association of } \\
\text { surgical } \\
\text { complications } \\
\text { with patients' } \\
\text { well-being } \\
\text { (yes/no/ } \\
\text { confounding) }\end{array}$ & $\begin{array}{l}\text { Types of } \\
\text { complications } \\
\text { and time points } \\
\text { of significant } \\
\text { effects }\end{array}$ & $\begin{array}{l}\text { Quality } \\
\text { assessment } \\
\text { score (out } \\
\text { of } 8 \text { ) }\end{array}$ \\
\hline Liu & 2010 & US & Primary & $\begin{array}{l}\mathrm{N}=679 / 1308 \\
\mathrm{Nc}(\text { early comps/ } \\
\text { anastomosis) }=54 \\
\mathrm{Nc}(\text { late comps/ } \\
\text { anastomosis) }=126 \\
\mathrm{Nc}(\text { early comps/ } \\
\text { anastomosis/rectal } \\
\text { cancer only) }=42 \\
\mathrm{Nc}(\text { late comps/ } \\
\text { ostomy/rectal cancer } \\
\text { only)=105 }\end{array}$ & $\begin{array}{l}\text { Patients with } \\
\text { long-term colorectal } \\
\text { cancer }\end{array}$ & $\begin{array}{l}\text { Observational, } \\
\text { cross-sectional }\end{array}$ & $\begin{array}{l}\text { Colorectal cancer } \\
\text { surgery }\end{array}$ & $\begin{array}{l}\text { Digestive, skin, genitourinary, } \\
\text { surgical, medical, immediate } \\
\text { indirect complicationsEarly } \\
\text { complications: those that } \\
\text { were first recorded within } \\
30 \text { days of the surgery. Late } \\
\text { complications: occurring } \\
31 \text { days after surgery/patient } \\
\text { computerised data }\end{array}$ & $\begin{array}{l}\text { QoL/ } 5-15 \text { years } \\
\text { postoperative/ } \\
\text { modified City of Hope } \\
\text { (mCOH)-QoL-Ostomy }\end{array}$ & Yes & $\begin{array}{l}\text { Enterocutaneous } \\
\text { fistula for all } \\
\text { patients and any } \\
\text { late } \\
\text { complications for } \\
\text { ostomy patients } \\
>5 \text { years } \\
\text { postsurgery }\end{array}$ & 6 \\
\hline Mentes & 2006 & Turkey & Primary & $\begin{array}{l}\mathrm{Nt} 1=253 / 302 \\
\mathrm{Nt} 2=244 \\
\mathrm{Nc}(\text { anal fistula/ } \\
\text { abscess) }=3 \\
\mathrm{Nc}(\mathrm{Fecal} \\
\text { Incontinence } \\
\text { Severity Index (FISI) } \\
>0)=7 \\
\mathrm{Nc}(\mathrm{FISI}, 0 \text { to }>4,21, \\
7)=3\end{array}$ & $\begin{array}{l}\text { Patients who } \\
\text { underwent lateral } \\
\text { internal } \\
\text { sphincterotomy (LIS) } \\
\text { for CAF }\end{array}$ & $\begin{array}{l}\text { Observational, } \\
\text { cohort, } \\
\text { prospective }\end{array}$ & $\begin{array}{l}\text { Lateral internal } \\
\text { sphincterotomy } \\
\text { (LIS) for CAF }\end{array}$ & $\begin{array}{l}\text { Anal incontinence/atient } \\
\text { examination+ FISI score }\end{array}$ & $\begin{array}{l}\text { QoL/preoperative } \\
\text { (admission) and } \\
12 \text { months } \\
\text { postoperative/GIQLI } \\
\text { and FIQL }\end{array}$ & $\begin{array}{l}\text { Unclear (due to } \\
\text { small number } \\
\text { of patients with } \\
\text { complications) }\end{array}$ & NA & 6 \\
\hline Pittman & 2008 & USA & Primary & $\begin{array}{l}\mathrm{N}=239 / 322 \\
\mathrm{Nc}=56\end{array}$ & $\begin{array}{l}\text { Veterans with an } \\
\text { ostomy after major } \\
\text { gastrointestinal } \\
\text { surgery requiring an } \\
\text { intestinal stoma }\end{array}$ & $\begin{array}{l}\text { Observational, } \\
\text { case-control, } \\
\text { cross-sectional }\end{array}$ & $\begin{array}{l}\text { Gastrointestinal } \\
\text { surgery requiring } \\
\text { an intestinal } \\
\text { stoma }\end{array}$ & $\begin{array}{l}\text { Ostomy complications: skin } \\
\text { problems, leakage and } \\
\text { difficulty with adjustment (ie, } \\
\text { leakage, peristomal irritant } \\
\text { dermitis, pain, bleeding, } \\
\text { stomal necrosis, prolapse, } \\
\text { stenosis, herniation, } \\
\text { retraction, infection, } \\
\text { mucotaneous separation, } \\
\text { difficulty adjusting)/patient } \\
\text { reports }\end{array}$ & $\begin{array}{l}\text { QoL/6 months } \\
\text { postoperative/ } \\
\text { mCOH-QoL-Ostomy }\end{array}$ & Yes & $\begin{array}{l}\text { Ostomy } \\
\text { complications } \\
\text { (skin problems, } \\
\text { leakage)/ } \\
6 \text { months } \\
\text { postsurgery }\end{array}$ & 6 \\
\hline Polese & 2012 & Italy & Primary & $\begin{array}{l}\mathrm{N}=147 / 211 \\
\mathrm{Nc}(\text { anastomotic } \\
\text { stenoses) }=22\end{array}$ & $\begin{array}{l}\text { Patients who } \\
\text { underwent elective } \\
\text { left colonic or rectal } \\
\text { resection and } \\
\text { colorectal } \\
\text { anastomosis for } \\
\text { neoplastic or } \\
\text { inflammatory } \\
\text { disease }\end{array}$ & $\begin{array}{l}\text { Observational, } \\
\text { cross-sectional }\end{array}$ & $\begin{array}{l}\text { Left colonic or } \\
\text { rectal resection } \\
\text { and colorectal } \\
\text { anastomosis }\end{array}$ & $\begin{array}{l}\text { Anastomotic stenosis/clinical } \\
\text { examination }\end{array}$ & $\begin{array}{l}\text { QoL/mean } 58 \text { (SD } \\
\pm 31 \text { ) months } \\
\text { postoperative/SF-36 }\end{array}$ & Yes & $\begin{array}{l}\text { Anastomotic } \\
\text { stenosis/58 (SD } \\
\pm 31 \text { ) months } \\
\text { postsurgery }\end{array}$ & 6 \\
\hline Rea & 2007 & USA & Primary & $\begin{array}{l}\mathrm{N} 1=505 / ? \\
\mathrm{Nt} 2=237\end{array}$ & $\begin{array}{l}\text { Patients who } \\
\text { underwent Roux-en- }\end{array}$ & & $\begin{array}{l}\text { LRYGB for } \\
\text { morbid obesity }\end{array}$ & & & Yes $^{\star}$ & $\begin{array}{l}\text { Complications } \\
\text { requiring }\end{array}$ & 6 \\
\hline
\end{tabular}

with patients' complications Quality

well-being and time points assessment

of 8 )

\begin{tabular}{ll} 
Study design & Type of surgery \\
\hline Observational, & Colorectal cancer
\end{tabular}

confound

Enterocutan

late

complications for

$>5$ years

nastomosis/rectal

were first recorded within

postsurgery

stomy/rectal cance

$\mathrm{Nt} 1=253 / 302$

$\mathrm{Nc}$ (anal fistula/

Patients who

Observational, Lateral internal

mputerised data

$\mathrm{Nc}$ (Fecal

intern

cohort,

sphincterotom

Anal incontinence/atient

12 months

Unclear (due to NA

f patients with

Incontinence

$>0$ ) $=7$

$\mathrm{Nc}$ (FISI, 0 to $>4,2$

P

ostomy after major

gastrointestinal

surgery requiring

case-control, Gurgery requirin

, leakage and

herniation,

underwent elective

left colonic or rectal

Continued 


\begin{tabular}{|c|c|c|c|c|c|c|c|c|c|c|c|c|}
\hline $\begin{array}{l}\text { First } \\
\text { author's } \\
\text { name }\end{array}$ & Year & Country & $\begin{array}{l}\text { Primary or } \\
\text { Secondary } \\
\text { aim }\end{array}$ & $\begin{array}{l}\text { Sample (N=number } \\
\text { of patients in } \\
\text { analysis/eligible } \\
\text { patients, } \mathrm{Nt}(\mathrm{i}) \\
\text { =sample size per } \\
\text { time point, } \\
\mathrm{Nc=patients} \mathrm{with} \\
\text { complications, } \\
\mathrm{N} 1=\text { cases vs } \\
\mathrm{N} 2=\text { controls) }\end{array}$ & $\begin{array}{l}\text { Patient inclusion } \\
\text { criteria }\end{array}$ & Study design & Type of surgery & $\begin{array}{l}\text { Surgical complications/ } \\
\text { method of recording }\end{array}$ & $\begin{array}{l}\text { Psychosocial } \\
\text { outcome/time } \\
\text { points/ } \\
\text { measurement tool }\end{array}$ & $\begin{array}{l}\text { Significant } \\
\text { association of } \\
\text { surgical } \\
\text { complications } \\
\text { with patients' } \\
\text { well-being } \\
\text { (yes/no/ } \\
\text { confounding) }\end{array}$ & $\begin{array}{l}\text { Types of } \\
\text { complications } \\
\text { and time points } \\
\text { of significant } \\
\text { effects }\end{array}$ & $\begin{array}{l}\text { Quality } \\
\text { assessment } \\
\text { score (out } \\
\text { of } 8 \text { ) }\end{array}$ \\
\hline & & & & $\begin{array}{l}\mathrm{Nt} 3=106 \\
\mathrm{Nc}(\mathrm{t} 2)=41 \\
\mathrm{Nc}(\mathrm{t} 3)=23\end{array}$ & $\begin{array}{l}\text { Y gastric bypass } \\
\text { (LRYGB) by one } \\
\text { surgeon for morbid } \\
\text { obesity }\end{array}$ & $\begin{array}{l}\text { Observational, } \\
\text { cohort, } \\
\text { prospective }\end{array}$ & $\begin{array}{l}\text { without } \\
\text { conversion to an } \\
\text { open procedure }\end{array}$ & $\begin{array}{l}\text { Postoperative complications } \\
\text { requiring intervention/method } \\
\text { not specified }\end{array}$ & $\begin{array}{l}\text { QoL/baseline, } 1 \text { and } \\
2 \text { years } \\
\text { postoperative/SF-36 }\end{array}$ & & $\begin{array}{l}\text { intervention } / 1 \\
\text { and } 2 \text { years } \\
\text { postsurgery }\end{array}$ & \\
\hline Riss & 2011 & Austria & Primary & $\begin{array}{l}\mathrm{N} 1=16 / 36 \text { (cases) } \\
\mathrm{N} 2=16 / \text { ? (controls) }\end{array}$ & $\begin{array}{l}\text { Cases: patients } \\
\text { operated for rectal } \\
\text { cancer and } \\
\text { developed } \\
\text { anastomotic leak. } \\
\text { Controls: patients } \\
\text { operated for rectal } \\
\text { cancer at the same } \\
\text { time period and had } \\
\text { an uneventful } \\
\text { postoperative course } \\
\text { matched by sex, age } \\
( \pm 5 \text { years), type of } \\
\text { resection, and } \\
\text { neoadjuvant therapy }\end{array}$ & $\begin{array}{l}\text { Observational, } \\
\text { case-control, } \\
\text { cross-sectional }\end{array}$ & $\begin{array}{l}\text { Rectal resection } \\
\text { for malignancies } \\
\text { on overall pelvic } \\
\text { organ function }\end{array}$ & $\begin{array}{l}\text { Anastomotic leakage: } \\
\text { defined as grade A (no } \\
\text { change in patient's } \\
\text { management), grade B } \\
\text { (requires active therapeutic } \\
\text { intervention but is managed } \\
\text { without relaparotomy) and } \\
\text { grade C (requires } \\
\text { relaparotomy)/review of the } \\
\text { institutional colorectal } \\
\text { database and individual } \\
\text { chart reviews }\end{array}$ & $\begin{array}{l}\text { QoL/106.8 months } \\
\text { postoperative (32.4- } \\
170.4) / S F-12\end{array}$ & No & NA & 7 \\
\hline Rutegard & 2008 & Sweden & Secondary & $\begin{array}{l}\mathrm{N}=355 / 446(79 \mathrm{a} 6 \%) \\
\mathrm{Nc}=56\end{array}$ & $\begin{array}{l}\text { Patients diagnosed } \\
\text { with an } \\
\text { oesophageal or } \\
\text { cardia cancer who } \\
\text { underwent } \\
\text { macroscopically } \\
\text { and microscopically } \\
\text { radical resection }\end{array}$ & $\begin{array}{l}\text { Observational, } \\
\text { cross-sectional }\end{array}$ & $\begin{array}{l}\text { Oesophageal } \\
\text { resection }\end{array}$ & $\begin{array}{l}\text { Technical surgical } \\
\text { complications, including } \\
\text { postoperative bleed } \\
\text { exceeding } 2000 \mathrm{ml} \text { or } \\
\text { requiring a reoperation, } \\
\text { anastomotic insufficiency, } \\
\text { necrosis of the substitute, } \\
\text { damage to the recurrent } \\
\text { nerve, thoracic duct damage } \\
\text { or gastric perforation/ } \\
\text { prospective scrutiny of } \\
\text { medical and } \\
\text { histopathological records, } \\
\text { operation charts, extensive } \\
\text { study protocol with } \\
\text { predefined exposure } \\
\text { alternatives }\end{array}$ & $\begin{array}{l}\text { QoL/6 months } \\
\text { postoperative/EORT } \\
\text { QLQ-C30, and } \\
\text { QLQ-OES1812 }\end{array}$ & Yes & $\begin{array}{l}\text { Technical } \\
\text { complications/ } \\
6 \text { months } \\
\text { postsurgery }\end{array}$ & 7 \\
\hline Scarpa & 2009 & Italy & Secondary & $\begin{array}{l}\mathrm{N}=47 / ? \\
\mathrm{Nc}=?\end{array}$ & $\begin{array}{l}\text { Patients admitted for } \\
\text { intestinal surgery for } \\
\text { Crohn's disease }\end{array}$ & $\begin{array}{l}\text { Observational, } \\
\text { cross-sectional }\end{array}$ & $\begin{array}{l}\text { Bowel resection } \\
\text { through midline } \\
\text { laparotomy or } \\
\text { with laparoscopic } \\
\text { assistance, end } \\
\text { ileostomy, } \\
\text { stricturoplasty }\end{array}$ & $\begin{array}{l}\text { Medical and surgical } \\
\text { complications and need of } \\
\text { reoperation ( } 2 \text { anastomotic } \\
\text { leaks, } 3 \text { intestinal } \\
\text { obstructions, } 2 \text { intestinal } \\
\text { bleeding, and a wound } \\
\text { infection were recorded and } \\
\text { two relaparotomies)/method } \\
\text { not specified }\end{array}$ & $\begin{array}{l}\text { QoL/3 months } \\
\text { postoperative/CGQLI }\end{array}$ & Confounding & $\begin{array}{l}\text { Any } \\
\text { complications/ } \\
3 \text { months } \\
\text { postsurgery }\end{array}$ & 3 \\
\hline Sharma & 2007 & UK & Secondary & & & & & & & Yes $^{*}$ & & 6 \\
\hline
\end{tabular}

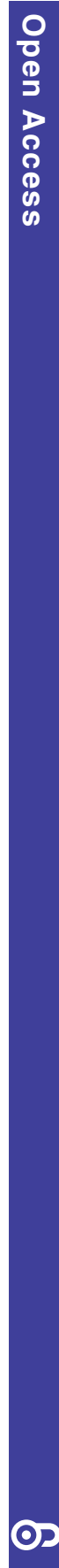

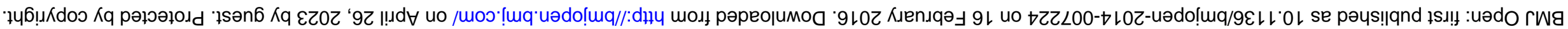




\begin{tabular}{|c|c|c|c|c|c|c|c|c|c|c|c|c|}
\hline $\begin{array}{l}\text { First } \\
\text { author's } \\
\text { name }\end{array}$ & Year & Country & $\begin{array}{l}\text { Primary or } \\
\text { Secondary } \\
\text { aim }\end{array}$ & $\begin{array}{l}\text { Sample (N=number } \\
\text { of patients in } \\
\text { analysis/eligible } \\
\text { patients, Nt(i) } \\
\text { =sample size per } \\
\text { time point, } \\
\mathrm{Nc}=\text { patients with } \\
\text { complications, } \\
\mathrm{N} 1=\text { cases vs } \\
\mathrm{N} 2=\text { controls) }\end{array}$ & $\begin{array}{l}\text { Patient inclusion } \\
\text { criteria }\end{array}$ & Study design & Type of surgery & $\begin{array}{l}\text { Surgical complications/ } \\
\text { method of recording }\end{array}$ & $\begin{array}{l}\text { Psychosocial } \\
\text { outcome/time } \\
\text { points/ } \\
\text { measurement tool }\end{array}$ & $\begin{array}{l}\text { Significant } \\
\text { association of } \\
\text { surgical } \\
\text { complications } \\
\text { with patients' } \\
\text { well-being } \\
\text { (yes/no/ } \\
\text { confounding) }\end{array}$ & $\begin{array}{l}\text { Types of } \\
\text { complications } \\
\text { and time points } \\
\text { of significant } \\
\text { effects }\end{array}$ & $\begin{array}{l}\text { Quality } \\
\text { assessment } \\
\text { score (out } \\
\text { of } 8 \text { ) }\end{array}$ \\
\hline & & & & $\begin{array}{l}\mathrm{Nt} 1=104 / 110 \\
\mathrm{Nt} 2=92 \\
\mathrm{Nc}=41\end{array}$ & $\begin{array}{l}\text { Consecutive patients } \\
\text { with newly } \\
\text { diagnosed colorectal } \\
\text { cancer scheduled } \\
\text { for elective open } \\
\text { resection in one } \\
\text { hospital trust }\end{array}$ & $\begin{array}{l}\text { Observational, } \\
\text { cohort, } \\
\text { prospective }\end{array}$ & $\begin{array}{l}\text { Elective resection } \\
\text { for colorectal } \\
\text { cancer }\end{array}$ & $\begin{array}{l}\text { Wound, urinary tract and } \\
\text { chest infections, cardiac and } \\
\text { respiratory complications, } \\
\text { deep venous thrombosis, } \\
\text { pulmonary embolism and } \\
\text { complications related to } \\
\text { anastomotic breakdown/ } \\
\text { method not specified }\end{array}$ & $\begin{array}{l}\text { QoL, anxiety, } \\
\text { depression, positive } \\
\text { vs negative } \\
\text { affectivity, mood } \\
\text { states/preoperative } \\
\text { (5-12 days } \\
\text { preoperative) and 6- } \\
8 \text { weeks } \\
\text { postoperative/ } \\
\text { FACT-C, EuroQOL } \\
\text { (EQ-5D), HADS, } \\
\text { PANAS, MRS }\end{array}$ & & $\begin{array}{l}\text { Complications } \\
\text { within } 30 \text { days of } \\
\text { operation/6- } \\
8 \text { weeks } \\
\text { postsurgery }\end{array}$ & \\
\hline Siassi & 2009 & Germany & Secondary & $\begin{array}{l}\mathrm{Nt} 1=93 / 113 \\
\mathrm{Nt} 2, \mathrm{t}=79 \\
\mathrm{Nc}=26\end{array}$ & $\begin{array}{l}\text { Patients undergoing } \\
\text { colorectal surgery } \\
\text { for benign and } \\
\text { malignant } \\
\text { disease }\end{array}$ & $\begin{array}{l}\text { Observational, } \\
\text { prospective, } \\
\text { cohort }\end{array}$ & $\begin{array}{l}\text { Resection of the } \\
\text { sigmoid } \\
\text { colon or rectum }\end{array}$ & $\begin{array}{l}\text { Postoperative complications } \\
\text { (anastomotic leak, wound } \\
\text { infection, delayed food } \\
\text { intake, fever, and bladder } \\
\text { dysfunction)/method not } \\
\text { specified }\end{array}$ & $\begin{array}{l}\text { QoL/preoperative, } 3 \\
\text { and } 12 \text { months } \\
\text { postoperative/SF-36 } \\
\text { and GLQI }\end{array}$ & Yes $^{*}$ & $\begin{array}{l}\text { Any } \\
\text { complications/ } \\
3 \text { months } \\
\text { postsurgery }\end{array}$ & 7 \\
\hline Targarona & 2004 & Spain & Primary & $\begin{array}{l}\mathrm{N}=37 / 46 \\
\mathrm{Nc}(\text { recurrent } \\
\text { hernias)=3 }\end{array}$ & $\begin{array}{l}\text { Patients diagnosed } \\
\text { with } \\
\text { paraoesophageal or } \\
\text { mixed hiatal hernia } \\
\text { (types II, III and IV) } \\
\text { with }>50 \% \text { of the } \\
\text { stomach in the chest }\end{array}$ & $\begin{array}{l}\text { Observational, } \\
\text { cross-sectional }\end{array}$ & $\begin{array}{l}\text { Laparoscopic } \\
\text { repair of } \\
\text { paraoesophageal } \\
\text { hiatal hernia }\end{array}$ & $\begin{array}{l}\text { Hernia recurrence (any } \\
\text { migration of the cardia to } \\
\text { chest level or evidence of a } \\
\text { new paraoesophageal sac)/a } \\
\text { barium swallow was given to } \\
\text { all patients to rule out an } \\
\text { anatomic recurrence. An } \\
\text { independent radiologist } \\
\text { evaluated all the explorations }\end{array}$ & $\begin{array}{l}\text { QoL/ } \geq 6 \text { months } \\
\text { postoperative } \\
\text { (median, 24; range, } \\
6-50 \text { )/SF-36, GDSS } \\
\text { and GIQLI }\end{array}$ & Yes & $\begin{array}{l}\text { Clinically } \\
\text { recurrent hernias/ } \\
\geq 6 \text { months } \\
\text { postsurgery }\end{array}$ & 5 \\
\hline Viklund & 2005 & Sweden & Secondary & $\begin{array}{l}\mathrm{N}=100 / 146 \\
\mathrm{Nc}=44\end{array}$ & $\begin{array}{l}\text { Patients newly } \\
\text { diagnosed with a } \\
\text { histologically verified } \\
\text { adenocarcinoma or } \\
\text { squamous-cell } \\
\text { carcinoma of the } \\
\text { oesophagus or } \\
\text { adenocarcinoma of } \\
\text { the gastric cardia } \\
\text { that underwent } \\
\text { macroscopically and } \\
\text { microscopically } \\
\text { radical tumour } \\
\text { resection }\end{array}$ & $\begin{array}{l}\text { Observational, } \\
\text { cross-sectional }\end{array}$ & $\begin{array}{l}\text { Oesophageal } \\
\text { resection surgery } \\
\text { for cancer }\end{array}$ & $\begin{array}{l}\text { Anastomotic leakage, } \\
\text { infections, respiratory } \\
\text { insufficiency, cardiac } \\
\text { complications, technical } \\
\text { complications, anastomotic } \\
\text { strictures, and others } \\
\text { (intervention needed to treat } \\
\text { embolus, deep venous } \\
\text { thrombosis, rupture of the } \\
\text { wound, intestinal obstruction, } \\
\text { stroke, renal failure, or liver } \\
\text { failure)/patient records }\end{array}$ & $\begin{array}{l}\text { QoL/6 months } \\
\text { postdischarge/ } \\
\text { QLQ-C30 and } \\
\text { OES-24 }\end{array}$ & Yes & $\begin{array}{l}\text { Any } \\
\text { complications, } \\
\text { anastomotic } \\
\text { leakage, } \\
\text { infection, } \\
\text { respiratory } \\
\text { insufficiency, } \\
\text { cardiac } \\
\text { complications, } \\
\text { technical } \\
\text { complications/ } \\
6 \text { months } \\
\text { postdischarge }\end{array}$ & 7 \\
\hline
\end{tabular}

Symptoms specific to oesophageal cancer. resection

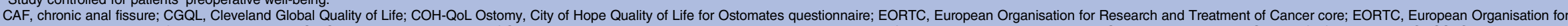
Research and Treatment of colorectal cancer; FACT-C, Functional Assessment of Cancer Therapy questionnaire with the colorectal module; FAP, familial adenomatous polyposis; FIQL, Fecal Incontinence Quality of Life Instrument; GDSS, Glasgow Dyspepsia Severity Score; GIQLI, Gastrointestinal Quality or Lile Index, GLQI, Gastrointestial Qually or Lile Index, HADS, Hosplial Anxily and Depression Scale, IBDQ, Ilammatory Bowel Disease Questionnaire, IPQ, Ingunal Pain Questionnaire; LRA, low rectal anastomosis; MRS, Mlood Rating Scale; NA, nof aval 


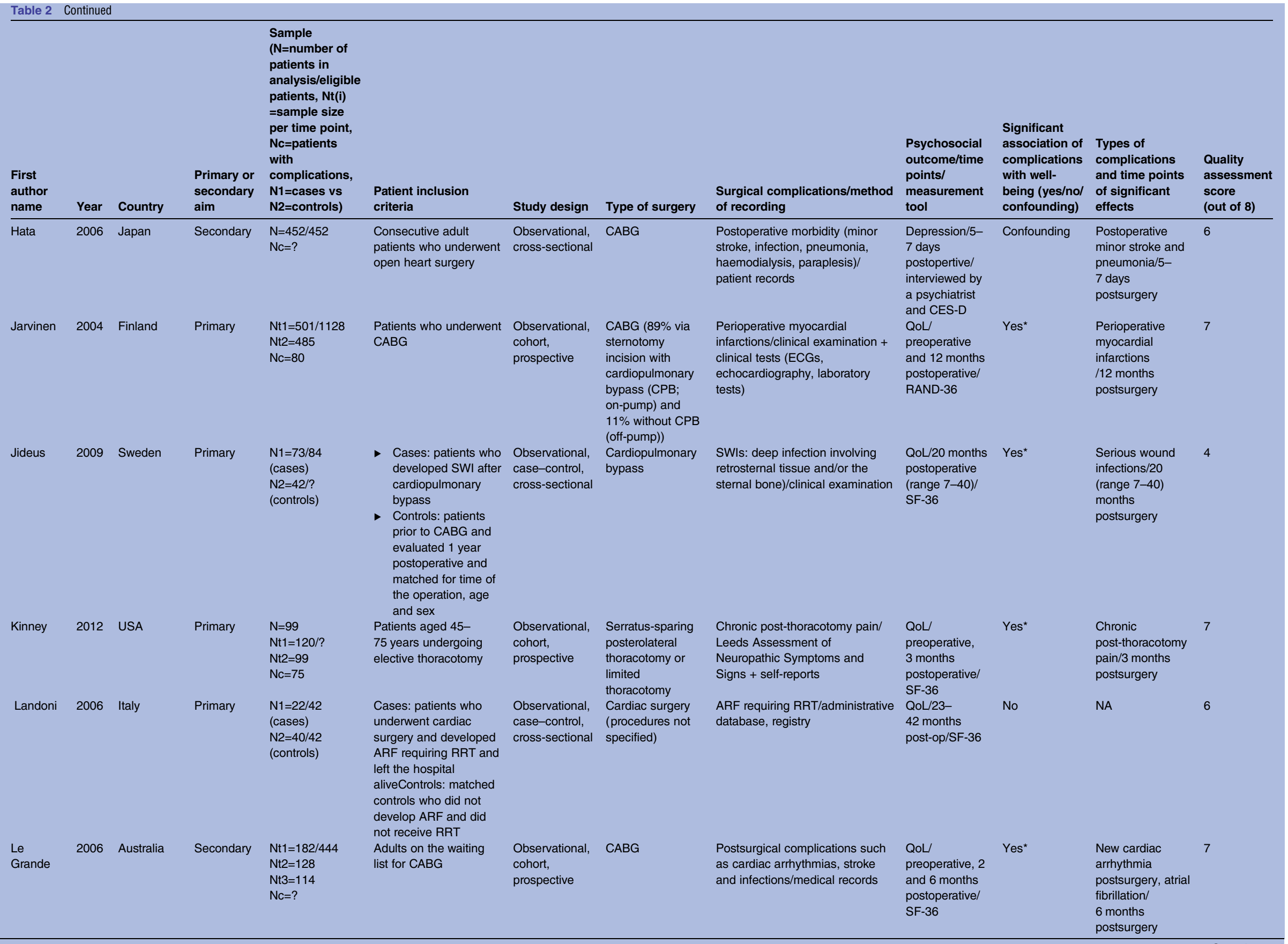




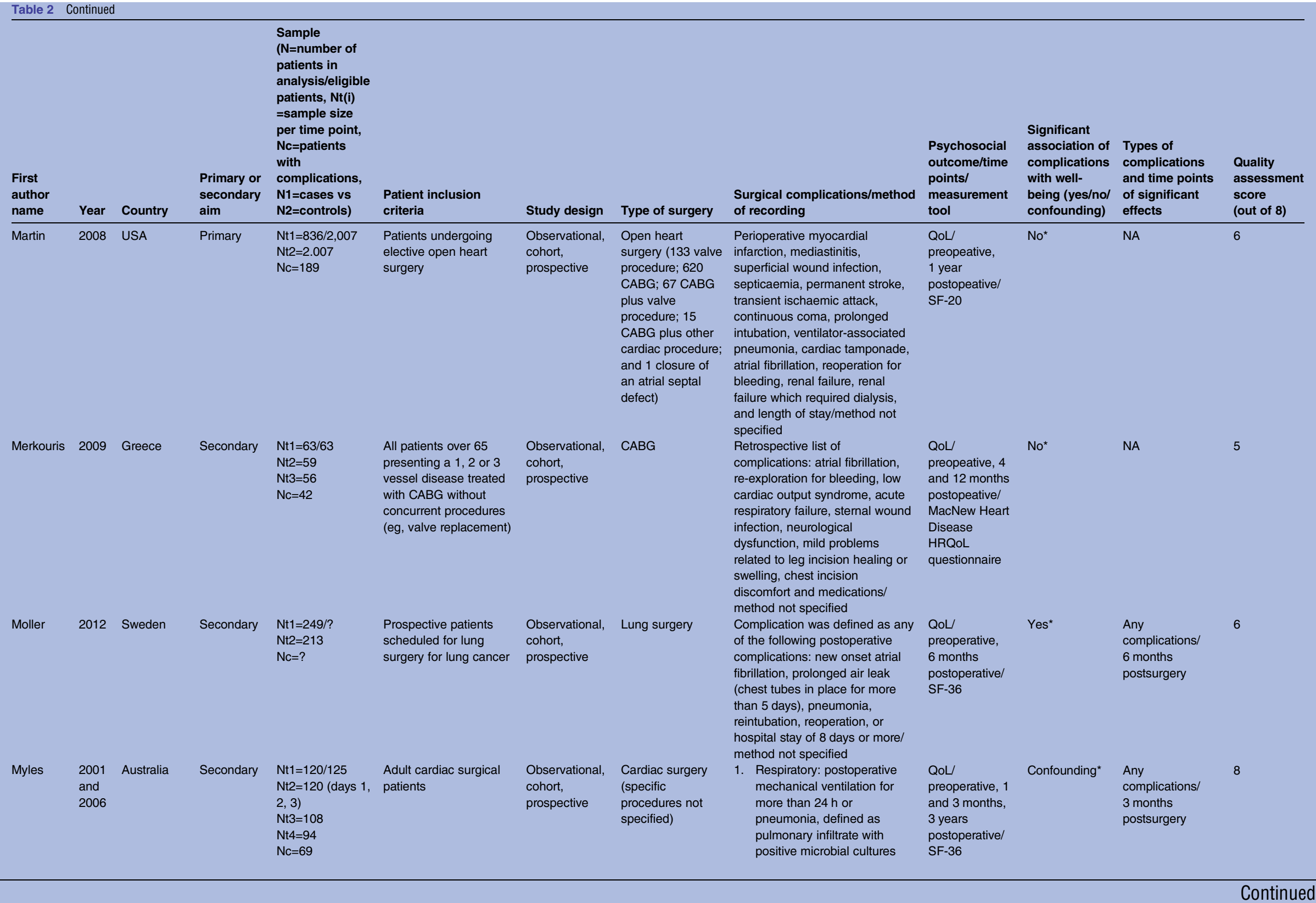

Continued 


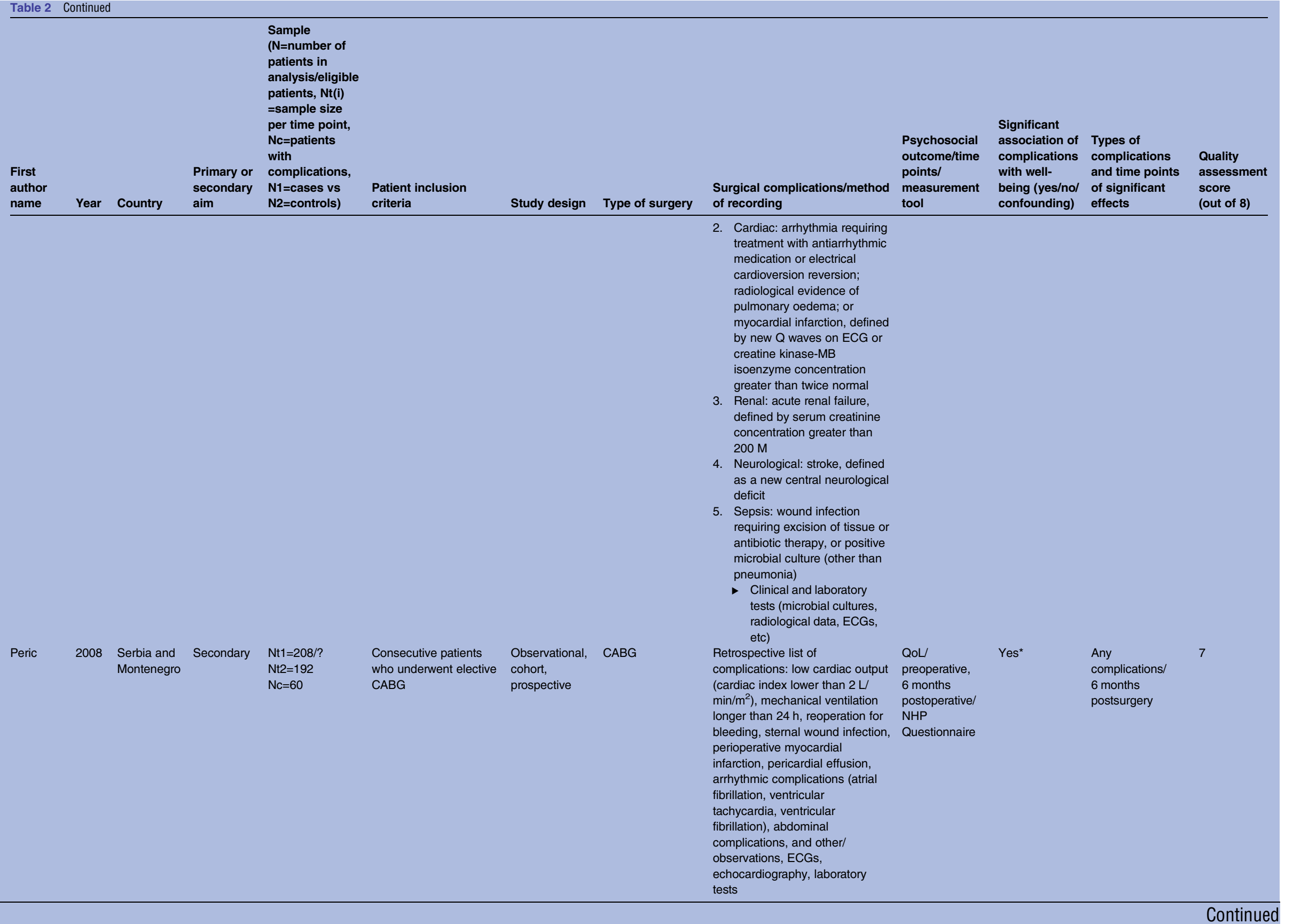




\begin{tabular}{|c|c|c|c|c|c|c|c|c|c|c|c|c|}
\hline $\begin{array}{l}\text { First } \\
\text { author } \\
\text { name }\end{array}$ & Year & Country & $\begin{array}{l}\text { Primary or } \\
\text { secondary } \\
\text { aim }\end{array}$ & $\begin{array}{l}\text { Sample } \\
\text { ( } \mathrm{N}=\text { number of } \\
\text { patients in } \\
\text { analysis/eligible } \\
\text { patients, Nt(i) } \\
\text { =sample size } \\
\text { per time point, } \\
\mathrm{Nc}=\text { patients } \\
\text { with } \\
\text { complications, } \\
\mathrm{N} 1=\text { cases vs } \\
\mathrm{N} 2=\text { controls) }\end{array}$ & $\begin{array}{l}\text { Patient inclusion } \\
\text { criteria }\end{array}$ & Study design & Type of surgery & $\begin{array}{l}\text { Surgical complications/method } \\
\text { of recording }\end{array}$ & $\begin{array}{l}\text { Psychosocial } \\
\text { outcome/time } \\
\text { points/ } \\
\text { measurement } \\
\text { tool }\end{array}$ & $\begin{array}{l}\text { Significant } \\
\text { association of } \\
\text { complications } \\
\text { with well- } \\
\text { being (yes/no/ } \\
\text { confounding) }\end{array}$ & $\begin{array}{l}\text { Types of } \\
\text { complications } \\
\text { and time points } \\
\text { of significant } \\
\text { effects }\end{array}$ & $\begin{array}{l}\text { Quality } \\
\text { assessment } \\
\text { score } \\
\text { (out of } 8 \text { ) }\end{array}$ \\
\hline Rodriguez & 2008 & USA & Secondary & $\begin{array}{l}\mathrm{N} t 1=397 / ? \\
\mathrm{Nt} 2=? \\
\mathrm{Nt} 3=? \\
\mathrm{Nt4}=? \\
\mathrm{Nc}=23\end{array}$ & $\begin{array}{l}\text { Patients diagnosed with } \\
\text { upper extremity } \\
\text { hyperhidrosis }(\mathrm{HH}) \\
\text { treated with thoracic } \\
\text { sympathectomy (TS) }\end{array}$ & $\begin{array}{l}\text { Observational, } \\
\text { cohort, } \\
\text { prospective }\end{array}$ & $\begin{array}{l}\text { Thoracoscopic } \\
\text { sympathectomy } \\
\text { for palmar and } \\
\text { axillary } \\
\text { hyperhidrosis }\end{array}$ & $\begin{array}{l}\text { CS: excessive sweating } \\
\text { considered abnormal in other } \\
\text { parts of the body after TS } \\
\text { Gustatory sweating: facial } \\
\text { sweating after eating foods } \\
\text { Excessive dryness: dryness } \\
\text { affecting the hands and } \\
\text { requiring hydration } \\
\text { - Method not specified }\end{array}$ & $\begin{array}{l}\text { QoL/ } \\
\text { preoperative, } \\
\text { discharge, } 6 \\
\text { and } 12 \text { months } \\
\text { postoperative/ } \\
\text { SF-36 }\end{array}$ & $\mathrm{No}^{*}$ & NA & 3 \\
\hline Tully & 2011 & Australia & Primary & $\begin{array}{l}\mathrm{N} t 1=226 / 238 \\
\mathrm{Nt} 2=222 \\
\mathrm{Nc}=56\end{array}$ & $\begin{array}{l}\text { Patients undergoing } \\
\text { first-time CABG surgery }\end{array}$ & $\begin{array}{l}\text { Observational, } \\
\text { cohort, } \\
\text { prospective }\end{array}$ & CABG & $\begin{array}{l}\text { New-onset AF between the } \\
\text { patient's day of admission to the } \\
\text { intensive care unit and the } \\
\text { median day of discharge (day 5) } \\
\text { after CABG during the index } \\
\text { hospitalisation/ECGs, } \\
\text { transthoracic echocardiographs } \\
\text { reviewed by technicians and } \\
\text { reviewers blinded to patients' } \\
\text { psychological distress scores }\end{array}$ & $\begin{array}{l}\text { Anxiety, } \\
\text { depression, } \\
\text { stress/ } \\
\text { preoperative } \\
\text { (mean=2 days, } \\
\mathrm{SD}=2 \text { days) } \\
\text { and } \\
\text { postoperative } \\
\text { (mean=6 days, } \\
\mathrm{SD}=2 \text { days)/ } \\
\mathrm{DASS}\end{array}$ & Yes $^{*}$ & $\begin{array}{l}\text { Atrial fibrillation/ } \\
6 \text { days } \\
\text { ( } \mathrm{SD}=2 \text { days }) \\
\text { postsurgery }\end{array}$ & 7 \\
\hline
\end{tabular}

*Study controlled for patients' preoperative well-being.

ARF, acute renal failure; AF, atrial fibrillation; BPI, Brief Pain Inventory; CES-D, Center for Epidemiological Studies Depression Scale; CS, compensatory sweating; DASS, Depression Anxiety

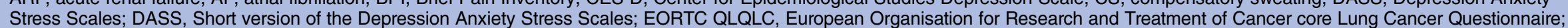

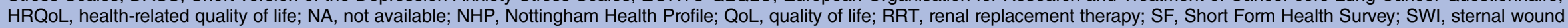
infection; T2DM, type 2 diabetes mellitus. 
Table 3 Key characteristics of studies in vascular surgery $(n=4)$

\begin{tabular}{|c|c|c|c|c|c|c|c|c|c|c|c|c|}
\hline $\begin{array}{l}\text { First } \\
\text { author } \\
\text { name }\end{array}$ & Year & Country & $\begin{array}{l}\text { Primary or } \\
\text { secondary } \\
\text { aim }\end{array}$ & $\begin{array}{l}\text { Sample ( } \mathrm{N}=\text { number } \\
\text { of patients in } \\
\text { analysis/eligible } \\
\text { patients, } \mathrm{Nt}(\mathrm{i}) \\
\text { =sample size per } \\
\text { time point, } \\
\mathrm{Nc}=\text { patients with } \\
\text { complications, } \\
\mathrm{N} 1=\text { cases vs } \\
\mathrm{N} 2=\text { controls) }\end{array}$ & Patient inclusion criteria & Study design & $\begin{array}{l}\text { Type of } \\
\text { surgery }\end{array}$ & $\begin{array}{l}\text { Surgical complications/ } \\
\text { method of recording }\end{array}$ & $\begin{array}{l}\text { Psychosocial } \\
\text { outcome and } \\
\text { time points }\end{array}$ & $\begin{array}{l}\text { Significant } \\
\text { association of } \\
\text { complications } \\
\text { with well- } \\
\text { being } \\
\text { (Yes/No/ } \\
\text { Confounding) }\end{array}$ & $\begin{array}{l}\text { Types of } \\
\text { complications } \\
\text { and } \\
\text { time-points of } \\
\text { significant } \\
\text { effects }\end{array}$ & $\begin{array}{l}\text { Quality } \\
\text { assessment } \\
\text { score } \\
\text { (out of } 8 \text { ) }\end{array}$ \\
\hline Lohse & 2009 & Germany & Secondary & $\begin{array}{l}\mathrm{N}=110 / 124 \\
\mathrm{NC}=?\end{array}$ & $\begin{array}{l}\text { Consecutive patients who } \\
\text { received a replacement of } \\
\text { the dilated ascending } \\
\text { aorta }\end{array}$ & $\begin{array}{l}\text { Observational, } \\
\text { cross-sectional }\end{array}$ & $\begin{array}{l}\text { Ascending } \\
\text { aorta } \\
\text { replacement }\end{array}$ & $\begin{array}{l}\text { Retrospective list: } \\
\text { postoperative bleeding, } \\
\text { myocardial infarction, stroke, } \\
\text { pneumonia, respiratory } \\
\text { insufficiency, acute renal } \\
\text { dysfunction, sepsis, lung } \\
\text { fistula/method not specified }\end{array}$ & $\begin{array}{l}\text { QoL/36.4 } \\
\pm 15.5 \text { months } \\
\text { postoperative } \\
\text { (11-58 months)/ } \\
\text { SF-36 }\end{array}$ & NO & NA & 4 \\
\hline Nguyen $^{\mathrm{a}}$ & 2007 & $\begin{array}{l}\text { USA and } \\
\text { Canada }\end{array}$ & Primary & $\begin{array}{l}\mathrm{Nt} 1=1296 / 1404 \\
\mathrm{Nt} 2=862 \\
\mathrm{~N} 3=732 \\
\mathrm{Nc}=543\end{array}$ & $\begin{array}{l}\text { Patients who underwent } \\
\text { lower extremity vein } \\
\text { bypass for CLI in } \\
\text { community and university } \\
\text { hospitals across the US } \\
\text { and Canada }\end{array}$ & $\begin{array}{l}\text { Observational, } \\
\text { cohort, } \\
\text { prospective }\end{array}$ & $\begin{array}{l}\text { Lower } \\
\text { extremity vein } \\
\text { bypass for } \\
\text { limb salvage in } \\
\text { CLI patients }\end{array}$ & $\begin{array}{l}\text { Wound complications (WC): } \\
\text { patients having infection, } \\
\text { necrosis, } \\
\text { hematoma-haemorrhage, or } \\
\text { seroma-lymphocele at the } \\
\text { surgical incision or harvest } \\
\text { site within } 30 \text { days of the } \\
\text { bypass surgery/Adverse } \\
\text { events clinical trial } \\
\text { documentation with reference } \\
\text { to source documentation } \\
\text { (hospital notes etc.) }\end{array}$ & $\begin{array}{l}\text { QoL/baseline, } 3 \\
\text { and } 12 \text { months } \\
\text { postoperative/ } \\
\text { VascuQol }\end{array}$ & Confounding* & $\begin{array}{l}\text { Wound } \\
\text { complications/ } \\
3 \text { months } \\
\text { postsurgery }\end{array}$ & 8 \\
\hline Nguyen ${ }^{\mathrm{b}}$ & 2006 & $\begin{array}{l}\text { USA and } \\
\text { Canada }\end{array}$ & Secondary & $\begin{array}{l}\mathrm{N} 1=1296 / 1404 \\
(92.3 \%) \\
\mathrm{N} 2=862(61.4 \%) \\
\mathrm{N} 3=732(52.1 \%) \\
\mathrm{Nc}=?\end{array}$ & $\begin{array}{l}\text { Patients who underwent } \\
\text { IB for CLI in community } \\
\text { and university hospitals } \\
\text { across the USA and } \\
\text { Canada }\end{array}$ & $\begin{array}{l}\text { Observational, } \\
\text { cohort, } \\
\text { prospective }\end{array}$ & $\begin{array}{l}\text { Infrainguinal } \\
\text { vein grafting } \\
\text { for limb } \\
\text { salvage in } \\
\text { patients with } \\
\text { CLI }\end{array}$ & $\begin{array}{l}\text { GREs: development of a } \\
>70 \% \text { graft stenosis or having } \\
\text { undergone a percutaneous or } \\
\text { surgical revision or a major } \\
\text { amputation/clinical tests } \\
\text { (angiography, } \\
\text { ultrasonography, etc), source } \\
\text { documentation (hospital } \\
\text { notes, discharge notes, } \\
\text { operative and procedural } \\
\text { notes, etc) }\end{array}$ & $\begin{array}{l}\text { QoL/ } \\
\text { preoperative, } 3 \\
\text { and } 12 \text { months } \\
\text { postoperative/ } \\
\text { VascuQol }\end{array}$ & Yes* & $\begin{array}{l}\text { GREs/ } \\
12 \text { months } \\
\text { postsurgery }\end{array}$ & 8 \\
\hline Subramonia & 2005 & UK & Primary & $\begin{array}{l}\mathrm{Nt} 1=70 / 70 \\
\mathrm{Nt} 2=59 \\
\mathrm{Nt} 3=62 \\
\mathrm{Nc}(\text { sensory } \\
\text { abnormalities) }=25 \\
\mathrm{Nc} \text { (bruising at } \mathrm{t} 1 \text { ) } \\
=58 \\
\mathrm{Nc} \text { (bruising at } \mathrm{t} 2 \text { ) } \\
=16\end{array}$ & $\begin{array}{l}\text { Patients with varicose } \\
\text { veins, either symptomatic } \\
\text { or with skin changes, } \\
\text { resulting from } \\
\text { incompetence of the } \\
\text { lesser saphenous vein } \\
\text { system (LSV) as } \\
\text { confirmed by handheld } \\
\text { Doppler examination or } \\
\text { duplex ultrasonography or } \\
\text { both and requiring } \\
\text { surgical intervention (both } \\
\text { day cases and inpatients) }\end{array}$ & $\begin{array}{l}\text { Observational, } \\
\text { cohort, } \\
\text { prospective }\end{array}$ & $\begin{array}{l}\text { Conventional } \\
\text { LSV stripping }\end{array}$ & $\begin{array}{l}\text { Bruising/tracing method } \\
\text { Sensory abnormalities, } \\
\text { both subjective } \\
\text { (paraesthesia and } \\
\text { dysaesthesia) and } \\
\text { objective/patient reports, } \\
\text { sensory testing }\end{array}$ & $\begin{array}{l}\text { QoL/ } \\
\text { preoperative, } \\
\text { discharge and } \\
6 \text { weeks } \\
\text { postoperative/ } \\
\text { Aberdeen } \\
\text { Varicose Vein } \\
\text { Questionnaire 2 }\end{array}$ & $\mathrm{No}^{*}$ & NA & 7 \\
\hline
\end{tabular}

*Study controlled for patients' preoperative well-being 
Table 4 Domains of patients' well-being that were significantly affected by surgical complications

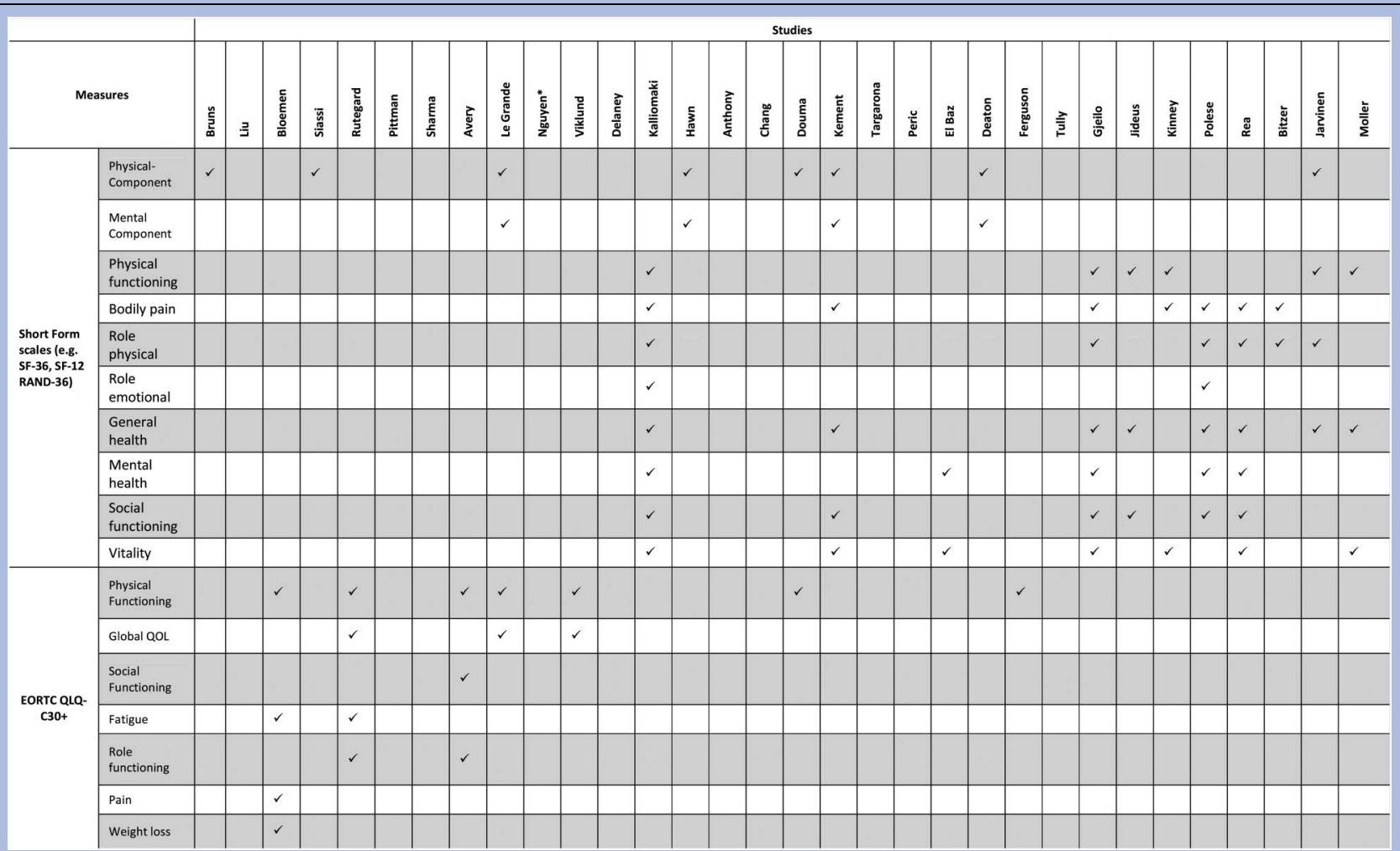




\section{Table 4 Continued}

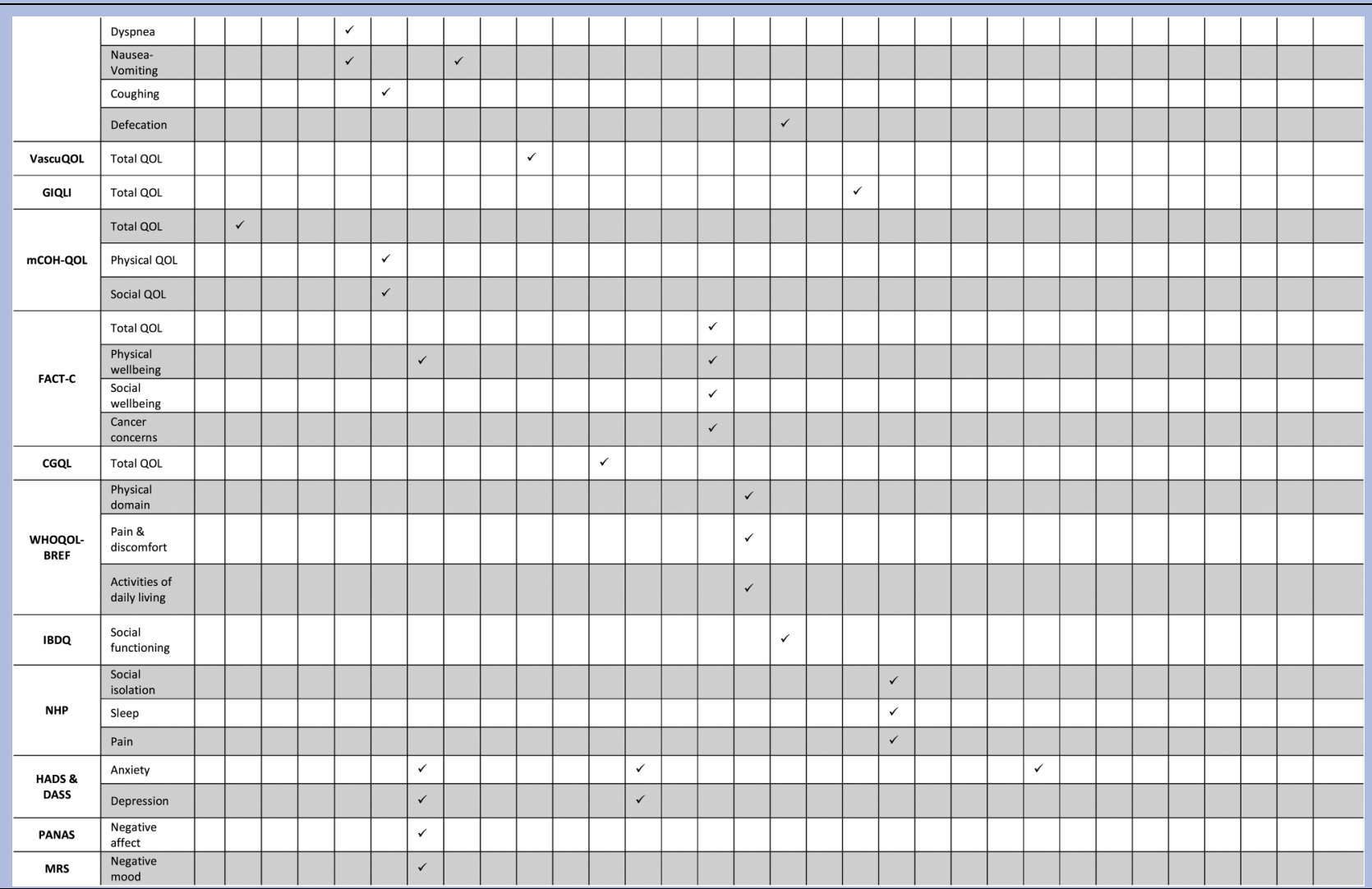

CGQL, Cleveland Global Quality of Life; DASS, Depression Anxiety Stress Scales; EORTC, European Organisation for Research and Treatment of Cancer; FACT-C, Functional Assessment of Cancer Therapy questionnaire with the colorectal module; GIQLI, Gastrointestinal Quality of Life Index; HADS, Hospital Anxiety and Depression Scale; IBDQ, Inflammatory Bowel Disease

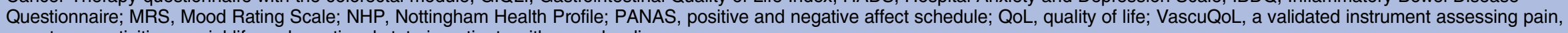
symptoms, activities, social life and emotional state in patients with vascular disease. 
tools with very different composite scores (eg, social, emotional and physical) was not considered valid. For that reason, only studies that used the SF scales ${ }^{67}$ were considered as they were the most commonly used QoL measures. Only three studies had sufficient data on the SF physical and mental QoL component scores. ${ }^{28} 3145$ The pooled mean differences (MD) between the two groups were statistically significant $(\mathrm{p}<0.001)$, indicating lower levels of physical ( $\mathrm{MD}=-3.28$, $\mathrm{CI}-4.71$ to -1.86 ) and mental (MD=-3.82, CI -4.97 to -2.67 ) QoL in patients who suffered complications compared with patients without complications. Two studies provided sufficient data for a meta-analysis on anxiety. ${ }^{30}{ }^{62}$ The pooled standardised MD was not significant $(p>0.05)$. A meta-analysis on depression was not possible as there was only one study with available data. ${ }^{30}$

For a more detailed report of the meta-analyses, see online supplementary materials $2-4$.

\section{The duration of the impact of surgical complications on patients' well-being}

Eighteen studies which reported significant associations of complications with postoperative psychosocial outcomes found a significant relationship of the presence of postoperative complications with worse psychosocial outcomes at 12 months postsurgery or later. ${ }^{16} 19-22$

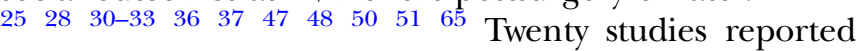
a significant association of complications with worse psychosocial outcomes at less than 12 months postsurgery. ${ }^{17} 18243539-464952545759606264$

\section{DISCUSSION}

This is, to our knowledge, the first systematic review of the literature investigating the impact of surgical complications on patients' psychosocial well-being. In line with our hypothesis, two-thirds of the included studies found a significant negative association between the occurrence of surgical complications and patients' postoperative well-being. The vast majority of those studies were of high quality. For instance, more than half of the studies with significant findings found that complications were an independent predictor of postoperative psychosocial outcomes after controlling for pre-existing differences on psychosocial outcomes, clinical and demographic variables.

Significant associations were reported in individual studies between surgical complications and lower scores on physical, emotional and social dimensions of the various QoL measures. A meta-analysis of three studies with sufficient QoL data collected with the SF scales suggests significant adverse effects of complications both on the physical and the mental health components. These findings are in agreement with earlier preliminary findings on the psychological burden that surgical adverse events often impose on patients. ${ }^{3}{ }^{4}$ Surgical complications were also significantly associated with higher postoperative anxiety and depression in individual studies, even though a population effect could not be shown due to the very small number of studies that measured the impact of surgical complications on anxiety and depression. Despite the fact that QoL is a useful screening outcome offering a general picture of a person's physical health and psychological state ${ }^{68}$ future studies on the psychosocial impact of surgical complications should also consider outcomes such as anxiety and depression as they offer a more accurate picture of a person's psychological well-being. Other relevant psychological outcomes such as post-traumatic stress, which was not measured in any of the included studies, would also be of relevance for future research in this area. It is also worth noting that strong conclusions cannot be drawn on the basis of the meta-analyses results due to the small number of studies included in them.

Complications that were found to significantly contribute to patients' low postoperative well-being ranged from severe adverse events such as anastomotic leaks after gastrointestinal surgery or perioperative myocardial infarctions after cardiac surgery to relatively minor complications such as wound infections or atrial fibrillation. It appears therefore that other than severe postoperative events, minor complications could also cause psychological distress during patients' recovery. For instance, wound complications could affect patients' satisfaction with their body image which could further compromise their QoL and psychological well-being. ${ }^{69}$ This finding potentially implies that the severity of complications as judged by healthcare professionals does not always correspond with patients' experience of complications. Moreover, complications were negatively associated with postoperative psychosocial outcomes not only after major surgical procedures but also after relatively minor operations, ${ }^{18} 28303143$ which suggests a potential independence of the magnitude of initial surgery with the effect of complications on patients' well-being. Further research on how complications affect patients' well-being after different types of surgery could help clarify this finding.

A number of studies also found a significant negative contribution of surgical complications to psychosocial outcomes more than 1 year postoperatively, suggesting that patients may suffer psychologically due to the experience of surgical complications for an extensive period of time after surgery. The above findings hold important implications for patients' recovery as there is growing evidence on the role of psychological stress in compromising the function of the immune system and slowing down wound healing. ${ }^{7-9}$ Surgical complications are likely to further prolong patients' recovery in almost a reciprocal cycle of distress and decreased immune function. The exact relationships between surgical complications, psychological distress and speed of recovery warrant further investigation.

It is noteworthy that a smaller number of studies did not find a significant association between complications and patients' postoperative psychosocial outcomes or found significant univariate associations which were not 
replicated in multivariate analyses. Even in studies showing a significant impact, there will be many patients who largely maintain their psychological health and QoL in the aftermath of complications. Other than clinical factors, patients' ways of coping with stress, their appraisals of surgery and their health, as well as their perceptions of support from their loved ones and healthcare professionals could explain the conditions under which complications affect patients' well-being, as suggested by wider literature on patients' adjustment after surgical treatment. ${ }^{70-72}$ The role of psychological factors as potential moderators of the psychological impact of surgical complications needs to be further explored.

Overall, the quality of the included studies was good as indicated by their relatively high-quality assessment scores and the small number of studies that scored exceptionally low. A substantial number of studies with significant findings controlled not only for patients' preoperative psychosocial outcomes but also for a variety of clinical and demographic factors confirming that surgical complications were an independent predictor of postoperative psychosocial outcomes above and beyond any pre-existing differences. The fact that the included studies used validated self-report measures for the measurement of psychosocial outcomes and the use of a very comprehensive search strategy also increase the validity of the findings.

\section{Limitations}

A few caveats should be borne in mind when interpreting the above findings. First, one-third of the studies did not define complications or did not describe the methods they used to record complications. Moreover, almost one-third of the studies did not provide information on response rates, which does not allow inferences about the representativeness of their samples.

Regarding the methodology of the systematic review, studies that were published before the year 2000 or with the majority of patients recruited before the year 2000 were excluded, albeit limiting this review to literature that was published in the last decade is expected to be more reflective of current surgical practice. It should also be noted that studies that were published past the final run of the search strategy (ie, May 2012) have not been considered. Caution should also be taken when interpreting these findings to other specialties as the clinical setting in which complications occur may affect their impact on patients' well-being. Another limitation was the very small number of studies with sufficient data for quantitative synthesis and the difficulty of synthesising data from different QoL measures, which resulted in restricting the meta-analyses on data collected only with the SF scales. The small number of studies with available data did not permit certain types of sensitivity analyses such as by surgical specialty, type of surgery (ie, minor vs major surgery) or underlying disease (eg, cancer vs other conditions), which could be significant determinants of the impact of complications on patients' well-being. Lastly, there is always the potential for publication bias where studies with significant results and big effect sizes are more easily published. ${ }^{73-75}$ It is worth adding that none of the included studies were randomised controlled trials due to the non-appropriateness of this design for the research questions that this review aims to answer.

\section{Implications of findings}

The results highlight the importance of considering patients' psychological needs in the aftermath of surgical complications. Surgical and nursing staff need to be aware of the challenges of surgical complications for patients' well-being and ensure that their psychological needs are not neglected. Screening patients who suffer postoperative complications for symptoms of psychological distress could help identify those patients who need psychological support. Facilitating patients' access to psychological support during and after their hospital stay could also be of great value for patients' postoperative well-being. For example, early referral to psychological services could prevent long-term psychological distress and may also mitigate the negative effects of stress on patients' recovery. Primary care practitioners and carers need to be aware of the psychological burden that surgical complications impose on patients in order to recognise their distress in time and to provide the support that patients need.

\section{CONCLUSIONS}

This is the first systematic review of the literature on the impact of surgical complications on patients' psychosocial well-being. The findings of this review suggest that surgical complications are potentially a significant independent predictor of patients' impaired postoperative psychosocial well-being often for a very long time postsurgery. It also appears that other than major complications, relatively minor adverse events may also compromise patients' psychosocial well-being, which implies that the clinical severity of complications may not always indicate how seriously patients will be affected by them. Patients who experience surgical complications report worse levels of different aspects of QoL than patients with uncomplicated recovery, often more than a year after their operation. The ways in which complications are managed (eg, reoperation vs conservative management), the type of surgery (eg, minor vs major), the underlying disease (eg, cancer vs other conditions), psychological factors (eg, patients' perceptions of support, illness perceptions, coping strategies) or cultural influences may be key moderators of the impact of surgical complications on patients' psychosocial well-being. Future research is needed on the contribution of the above factors on the impact of surgical complications on psychological outcomes such as anxiety, depression and post-traumatic stress, as well as on how to support patients who experience a complicated postoperative recovery. 
Contributors AP, OF, RD, AA and CV contributed to the conception and design of this review, and reviewed drafts of the manuscript. AP also screened all the articles retrieved by the literature searches, extracted and synthesised the data of the eligible for inclusion articles, appraised the study quality of the included articles and wrote the initial draft of this manuscript. RD screened a sample of the retrieved articles at title, abstract and full text, and AA extracted data from and scored the quality of a sample of the included articles.

Funding This work was supported by funding from the Health Foundation and the National Institute for Health Research (NIHR) Imperial Patient Safety Translational Research Centre.

Disclaimer The views expressed are those of the author(s) and not necessarily those of the funders.

Competing interests None declared

Provenance and peer review Not commissioned; externally peer reviewed.

Data sharing statement No additional data are available.

Open Access This is an Open Access article distributed in accordance with the terms of the Creative Commons Attribution (CC BY 4.0) license, which permits others to distribute, remix, adapt and build upon this work, for commercial use, provided the original work is properly cited. See: http:// creativecommons.org/licenses/by/4.0/

\section{REFERENCES}

1. Dindo D, Demartines N, Clavien PA. Classification of surgical complications: a new proposal with evaluation in a cohort of 6336 patients and results of a survey. Ann Surg 2004;240:205-13.

2. Tevis SE, Kennedy GD. Postoperative complications and implications on patient-centered outcomes. J Surg Res 2013:181:106-13

3. Vincent CA, Pincus T, Scurr JH. Patients' experience of surgical accidents. Qual Health Care 1993;2:77-82.

4. Gardner G, Elliott D, Gill J, et al. Patient experiences following cardiothoracic surgery: an interview study. Eur J Cardiovasc Nur 2005:4:242-50.

5. Walburn J, Vedhara K, Hankins M, et al. Psychological stress and wound healing in humans: a systematic review and meta-analysis. $J$ Psychosom Res 2009;67:253-71.

6. Ebrecht M, Hextall J, Kirtley LG, et al. Perceived stress and cortisol levels predict speed of wound healing in healthy male adults. Psychoneuroendocrinology 2004;29:798-809.

7. Herbert TB, Cohen S. Stress and immunity in humans: a meta-analytic review. Psychosom Med 1993:55:364-79.

8. Kiecolt-Glaser JK, McGuire L, Robles TF, et al. Psychoneuroimmunology: psychological influences on immune function and health. J Consult Clin Psych 2002;70:537-47.

9. Kiecolt-Glaser JK, Page GG, Marucha PT, et al. Psychological influences on surgical recovery. Perspectives from psychoneuroimmunology. Am Psychol 1998;53:1209-18.

10. Yehuda R. Post-traumatic stress disorder. N Engl J Med 2002;346:108-14.

11. Gawande AA, Thomas EJ, Zinner MJ, et al. The incidence and nature of surgical adverse events in Colorado and Utah in 1992 Surgery 1999;126:66-75.

12. Wells GA, Shea B, O'Connell D, et al. The Newcastle-Ottawa Scale (NOS) for assessing the quality if nonrandomized studies in meta-analyses. http://www.ohri.ca/programs/clinical_epidemiology/ oxford.asp (accessed 22 May 2014).

13. The Cochrane Collaboration. Review Manager (RevMan). Version 5.2 ed. Copenhagen: The Nordic Cochrane Centre, 2012.

14. Higgins J, Green S. Cochrane Handbook for Systematic Reviews of Interventions. 2011. http://www.cochrane-handbook.org (accessed 22 May 2014).

15. Field AP, Gillett R. How to do a meta-analysis. Br J Math Stat Psychol 2010;63:665-94.

16. Anthony T, Long J, Hynan LS, et al. Surgical complications exert a lasting effect on disease-specific health-related quality of life for patients with colorectal cancer. Surgery 2003;134:119-25.

17. Avery KNL, Metcalfe C, Nicklin J, et al. Satisfaction with care: an independent outcome measure in surgical oncology. Ann Surg Oncol 2006;13:817-22

18. Bitzer EM, Lorenz C, Nickel S, et al. Assessing patient-reported outcomes of cholecystectomy in short-stay surgery. Surg Endosc 2008;22:2712-19.
19. Bloemen JG, Visschers RGJ, Truin W, et al. Long-term quality of life in patients with rectal cancer: association with severe postoperative complications and presence of a stoma. Dis Colon Rectum 2009:52:1251-8.

20. Bruns H, Kratschmer K, Hinz U, et al. Quality of life after curative liver resection: a single center analysis. World $J$ Gastroenterol 2010;16:2388-95.

21. Champault A, Duwat O, Polliand C, et al. Quality of life after laparoscopic gastric banding: prospective study (152 cases) with a follow-up of 2 years. Surg Laparosc Endosc Percutan 2006;16:131-6.

22. Chang CY, Huang CK, Chang YY, et al. Prospective study of health-related quality of life after Roux-en-Y bypass surgery for morbid obesity. Br J Surg 2010;97:1541-6.

23. Dasgupta D, Smith AB, Hamilton-Burke W, et al. Quality of life after liver resection for hepatobiliary malignancies. $\mathrm{Br} J$ Surg 2008;95:845-54.

24. Delaney CP, Kiran RP, Senagore AJ, et al. Quality of life improves within 30 days of surgery for Crohn's disease. J Am Coll Surg 2003;196:714-21.

25. Douma KFL, Bleiker EMA, Vasen HFA, et al. Quality of life and consequences for daily life of familial adenomatous polyposis (FAP) family members. Colorectal Dis 2011;13:669-77.

26. Dubernard G, Piketty M, Rouzier R, et al. Quality of life after laparoscopic colorectal resection for endometriosis. Hum Reprod 2006;21:1243-7.

27. El-Awady SE, Elkholy AAM. Beneficial effect of inguinal hernioplasty on testicular perfusion and sexual function. Hernia 2009:13:251-8.

28. Hawn MT, Itani KM, Giobbie-Hurder A, et al. Patient-reported outcomes after inguinal herniorrhaphy. Surgery 2006;140:198-205

29. Ince M, Kirat HT, Geisler DP, et al. The negative effects of surgery persist beyond the early postoperative period after laparoscopic colorectal resection. Tech Coloproctol 2011:15:173-7.

30. Kalliomaki ML, Sandblom G, Gunnarsson U, et al. Persistent pain after groin hernia surgery: a qualitative analysis of pain and its consequences for quality of life. Acta Anaesthesiol Scand 2009;53:236-46.

31. Kement M, Karabulut M, Gezen FC, et al. Mild and severe ana incontinence after lateral internal sphincterotomy: risk factors, postoperative anatomical findings and quality of life. Eur Surg Res 2011:47:26-31.

32. Lim M, Akhtar S, Sasapu K, et al. Clinical and subclinical leaks after low colorectal anastomosis: a clinical and radiologic study. Dis Colon Rectum 2006:49:1611-19.

33. Liu L, Herrinton LJ, Hornbrook MC, et al. Early and late complications among long-term colorectal cancer survivors with ostomy or anastomosis. Dis Colon Rectum 2010;53:200-12.

34. Mentes BB, Tezcaner T, Yilmaz U, et al. Results of lateral internal sphincterotomy for chronic anal fissure with particular reference to quality of life. Dis Colon Rectum 2006;49:1045-51.

35. Pittman J, Rawl SM, Schmidt CM, et al. Demographic and clinical factors related to ostomy complications and quality of life in veterans with an ostomy. J Wound Ostomy Continence 2008;35:493-503.

36. Polese L, Vecchiato M, Frigo AC, et al. Risk factors for colorectal anastomotic stenoses and their impact on quality of life: What are the lessons to learn? Colorectal Dis 2012;14:e124-8.

37. Rea JD, Yarbrough DE, Leeth RR, et al. Influence of complications and extent of weight loss on quality of life after laparoscopic Roux-en-Y gastric bypass. Surg Endosc 2007;21:1095-100.

38. Riss S, Stremitzer S, Riss K, et al. Pelvic organ function and quality of life after anastomotic leakage following rectal cancer surgery. Wien Klin Wochenschr 2011;123:53-7.

39. Rutegard M, Lagergren J, Rouvelas I, et al. Population-based study of surgical factors in relation to health-related quality of life after oesophageal cancer resection. Br J Surg 2008;95:592-601.

40. Scarpa M, Ruffolo C, Bassi D, et al. Intestinal surgery for Crohn's disease: predictors of recovery, quality of life, and costs. $J$ Gastrointest Surg 2009;13:2128-35.

41. Sharma A, Sharp DM, Walker LG, et al. Predictors of early postoperative quality of life after elective resection for colorectal cancer. Ann Surg Oncol 2007;14:3435-42.

42. Siassi $M$, Weiss $M$, Hohenberger $W$, et al. Personality rather than clinical variables determines quality of life after major colorectal surgery. Dis Colon Rectum 2009;52:662-8.

43. Targarona EM, Novell J, Vela S, et al. Mid term analysis of safety and quality of life after the laparoscopic repair of paraesophageal hiatal hernia. Surg Endosc 2004;18:1045-50.

44. Viklund $P$, Lindblad M, Lagergren J. Influence of surgery-related factors on quality of life after esophageal or cardia cancer resection. World J Surg 2005;29:841-8. 
45. Deaton C, Thourani V. Patients with type 2 diabetes undergoing coronary artery bypass graft surgery: predictors of outcomes. Eur $J$ Cardiovasc Nurs 2009;8:48-56.

46. El Baz N, Middel B, van Dijk JP, et al. EuroSCORE predicts poor health-related physical functioning six month postcoronary artery bypass graft surgery. J Cardiovasc Surg 2008;49:663-72.

47. Ferguson MK, Parma CM, Celauro AD, et al. Quality of life and mood in older patients after major lung resection. Ann Thorac Surg 2009;87:1007-12; discussion 1012-13.

48. Gjeilo KH, Klepstad P, Wahba A, et al. Chronic pain after cardiac surgery: a prospective study. Acta Anaesthesiol Scand 2010;54:70-8.

49. Hata M, Yagi Y, Sezai A, et al. Risk analysis for depression and patient prognosis after open heart surgery. Circ J 2006; 70: 389-92.

50. Jarvinen O, Julkunen J, Saarinen T, et al. Perioperative myocardial infarction has negative impact on health-related quality of life following coronary artery bypass graft surgery. Eur $\mathrm{J}$ Cardiothorac 2004;26:621-7.

51. Jideus L, Liss A, Stahle E. Patients with sternal wound infection after cardiac surgery do not improve their quality of life. Scand Cardiovasc J 2009;43:194-200.

52. Kinney MAO, Hooten WM, Cassivi SD, et al. Chronic postthoracotomy pain and health-related quality of life. Ann Thorac Surg 2012;93:1242-7.

53. Landoni G, Zangrillo A, Franco A, et al. Long-term outcome of patients who require renal replacement therapy after cardiac surgery. Eur J Anaesth 2006;23:17-22.

54. Le Grande MR, Elliott PC, Murphy BM, et al. Health related quality of life trajectories and predictors following coronary artery bypass surgery. Health Qual Life Outcomes 2006:4:49.

55. Martin LM, Halpin LS, Barnett SD, et al. The association between early outcome, health-related quality of life, and survival following elective open-heart surgery. J Cardiovasc Nurs 2008;23:432-42.

56. Merkouris A, Apostolakis E, Pistolas D, et al. Quality of life after coronary artery bypass graft surgery in the elderly. Eur $\mathrm{J}$ Cardiovasc Nurs 2009;8:74-81.

57. Moller A, Sartipy U. Predictors of postoperative quality of life after surgery for lung cancer. J Thorac Oncol 2012;7:406-11.

58. Myles PS. Quality of life at three years after cardiac surgery: relationship with preoperative status and quality of recovery. Anaesth Intensive Care 2006;34:176.

59. Myles PS, Hunt JO, Fletcher $\mathrm{H}$, et al. Relation between quality of recovery in hospital and quality of life at 3 months after cardiac surgery. Anesthesiology 2001;95:862-7.
60. Peric V, Borzanovic M, Stolic R, et al. Predictors of worsening of patients' quality of life six months after coronary artery bypass surgery. J Cardiac Surg 2008;23:648-54.

61. Rodriguez PM, Freixinet JL, Hussein M, et al. Side effects, complications and outcome of thoracoscopic sympathectomy for palmar and axillary hyperhidrosis in 406 patients. Eur $J$ Cardiothorac 2008:34:514-19.

62. Tully PJ, Bennetts JS, Baker RA, et al. Anxiety, depression, and stress as risk factors for atrial fibrillation after cardiac surgery. Heart Lung 2011;40:4-11.

63. Lohse F, Lang N, Schiller W, et al. Quality of life after replacement of the ascending aorta in patients with true aneurysms. Tex Heart Inst J 2009;36:104-10.

64. Nguyen LL, Brahmanandam S, Bandyk DF, et al. Female gender and oral anticoagulants are associated with wound complications in lower extremity vein bypass: an analysis of 1404 operations for critical limb ischemia. J Vasc Surg 2007;46:1191-7.

65. Nguyen LL, Moneta GL, Conte MS, et al. Prospective multicenter study of quality of life before and after lower extremity vein bypass in 1404 patients with critical limb ischemia. J Vasc Surg 2006;44:977-83; discussion 983-4.

66. Subramonia S, Lees T. Sensory abnormalities and bruising after long saphenous vein stripping: impact on short-term quality of life. $J$ Vasc Surg 2005;42:510-14.

67. Ware JE, Kosinski M. SF-36 physical \& mental health summary scales: a manual for users of version 1. Quality Metric Inc, 2001.

68. WHOQOL. Development of the WHOQOL: rationale and current status. Int J Ment Health 1994;23:24-56.

69. Dunker MS, Stiggelbout AM, van Hogezand RA, et al. Cosmesis and body image after laparoscopic-assisted and open ileocolic resection for Crohn's disease. Surg Endosc 1998;12:1334-40.

70. Boehmer S, Luszczynska A, Schwarzer R. Coping and quality of life after tumor surgery: personal and social resources promote different domains of quality of life. Anxiety Stress Coping 2007;20:61-75.

71. Kulik JA, Mahler HI. Social support and recovery from surgery. Health Psychol 1989;8:221-38.

72. Orbell S, Johnston M, Rowley D, et al. Cognitive representations of illness and functional and affective adjustment following surgery for osteoarthritis. Soc Sci Med 1998;47:93-102.

73. Easterbrook PJ, Gopalan R, Berlin JA, et al. Publication bias in clinical research. Lancet 1991;337:867-72.

74. Dickersin KAY, Min YI. Publication bias: the problem that won't go away. Ann N Y Acad Sci 1993;703:135-46; discussion 146-8.

75. Dwan K, Altman DG, Arnaiz JA, et al. Systematic review of the empirical evidence of study publication bias and outcome reporting bias. PLOS ONE 2008;3:e3081. 